Review

\title{
Assessment of the Association of COPD and Asthma with In-Hospital Mortality in Patients with COVID-19. A Systematic Review, Meta-Analysis, and Meta-Regression Analysis
}

\author{
Felix M. Reyes ${ }^{1,2} \mathbb{D}^{-}$, Manuel Hache-Marliere ${ }^{2,3, *}$, Dimitris Karamanis ${ }^{4}$, Cesar G. Berto ${ }^{2,3}$, Rodolfo Estrada ${ }^{5}$, \\ Matthew Langston ${ }^{2,3}$, George Ntaios ${ }^{6}{ }^{(}$, Perminder Gulani ${ }^{2,3}$, Chirag D. Shah ${ }^{1,2}$ and Leonidas Palaiodimos ${ }^{2,7}$ \\ 1 Division of Pulmonary Medicine, Montefiore Medical Center, Bronx, NY 10461, USA; \\ freyesv@montefiore.org (F.M.R.); cshah@montefiore.org (C.D.S.) \\ 2 Albert Einstein College of Medicine, Bronx, NY 10461, USA; bertomc@nychhc.org (C.G.B.); \\ matthew.langston@nychhc.org (M.L.); perminder.gulani@nychhc.org (P.G.); \\ leonidas.palaiodimos@nychhc.org (L.P.) \\ 3 Department of Medicine, Jacobi Medical Center, Bronx, NY 10461, USA \\ 4 Department of Economics, University of Piraeus, 18534 Attica, Greece; dkaramanis@hotmail.com \\ 5 Division of Pulmonary Diseases and Critical Care Medicine, University of Texas Health at San Antonio, \\ San Antonio, TX 78229, USA; rodolfo_estrada1@yahoo.com \\ 6 Department of Internal Medicine, Faculty of Medicine, School of Health Sciences, University of Thessaly, \\ 38221 Larissa, Greece; gntaios@med.uth.gr \\ 7 Division of Hospital Medicine, Jacobi Medical Center, Bronx, NY 10461, USA \\ * Correspondence: hachem@nychhc.org
}

Citation: Reyes, F.M.;

Hache-Marliere, M.; Karamanis, D.; Berto, C.G.; Estrada, R.; Langston, M.; Ntaios, G.; Gulani, P.; Shah, C.D.; Palaiodimos, L. Assessment of the Association of COPD and Asthma with In-Hospital Mortality in Patients with COVID-19. A Systematic Review, Meta-Analysis, and Meta-Regression Analysis. J. Clin. Med. 2021, 10, 2087. https://doi.org/10.3390/jcm10102087

Academic Editor: Arschang Valipour

Received: 6 March 2021

Accepted: 23 April 2021

Published: 13 May 2021

Publisher's Note: MDPI stays neutral with regard to jurisdictional claims in published maps and institutional affiliations.

Copyright: (C) 2021 by the authors Licensee MDPI, Basel, Switzerland. This article is an open access article distributed under the terms and conditions of the Creative Commons Attribution (CC BY) license (https:/ / creativecommons.org/licenses/by/ $4.0 /)$.
Abstract: Together, chronic obstructive pulmonary disease (COPD) and asthma account for the most common non-infectious respiratory pathologies. Conflicting preliminary studies have shown varied effect for COPD and asthma as prognostic factors for mortality in coronavirus disease 2019 (COVID-19). The aim of this study was to explore the association of COPD and asthma with inhospital mortality in patients with COVID-19 by systematically reviewing and synthesizing with a meta-analysis the available observational studies. MEDLINE, Scopus, and medRxiv databases were reviewed. A random-effects model meta-analysis was used, and I-square was utilized to assess for heterogeneity. In-hospital mortality was defined as the primary endpoint. Sensitivity and meta-regression analyses were performed. Thirty studies with 21,309 patients were included in this meta-analysis (1465 with COPD and 633 with asthma). Hospitalized COVID-19 patients with COPD had higher risk of death compared to those without COPD (OR: 2.29; 95\% CI: 1.79-2.93; I² 59.6\%). No significant difference in in-hospital mortality was seen in patients with and without asthma (OR: 0.87; 95\% CI: 0.68-1.10; $\mathrm{I}^{2} 0.0 \%$ ). The likelihood of death was significantly higher in patients with COPD that were hospitalized with COVID-19 compared to patients without COPD. Further studies are needed to assess whether this association is independent or not. No significant difference was demonstrated in COVID-19-related mortality between patients with and without asthma.

Keywords: chronic obstructive pulmonary disease (COPD); asthma; COVID-19; meta-analysis; mortality

\section{Introduction}

Together, chronic obstructive pulmonary disease (COPD) and asthma account for the most common non-infectious respiratory pathologies [1-3]. COPD represents the fourth cause of death in the world and is characterized by an irreversible airway obstruction caused by a mix of airway inflammation and parenchymal destruction. This happens commonly after exposure to particles or gases, most commonly associated with smoking [4]. The World Health Organization (WHO) estimates a worldwide prevalence of COPD at about 265 million people, and the general prevalence of moderate to severe COPD in 
65 million people [1]. Asthma is characterized by chronic airway inflammation that causes reversible obstruction of airflow [5]. The WHO estimates that approximately 339 million people live with asthma worldwide, and that almost 500,000 yearly deaths can be attributed to asthma [1].

Respiratory viral infections are an important cause of asthma and COPD exacerbations [6-8]. Coronaviruses are frequently listed as pathogens found in the airway of COPD patients during exacerbations [9-11]. In a systematic review involving 1728 patients, coronaviruses were found in $4 \%$ of patients with COPD exacerbations [12]. Likewise, coronavirus infections seem to be involved in 2 to $8 \%$ of acute asthma exacerbations [13-17].

Coronavirus Disease 2019 (COVID-19), a disease caused by infection with severe acute respiratory syndrome coronavirus 2 (SARS-CoV-2), reached the level of pandemic rapidly and caused almost 3 million deaths worldwide within a year (WHO) [18]. Given the facts that COPD and asthma have high prevalence and morbidity and COVID-19 has mainly manifestations from the respiratory system, we hypothesized that patients with COPD and Asthma are at increased risk of death when hospitalized due to COVID-19. Thus, we aimed to systematically review and synthesize with a meta-analysis of available studies the mortality effect of asthma and COPD among hospitalized patients with COVID-19.

\section{Materials and Methods}

This systematic review and meta-analysis was performed following Preferred Reporting Items for Systematic reviews and Meta-analysis (PRISMA) guidelines (Appendix A Table A1) [19].

\subsection{Literature Search}

We conducted a systematic literature search of Medline (U.S. National Library of Medicine, Bethesda, MD, USA), Scopus (Elsevier, Amsterdam, Netherlands) and medRxiv databases (Cold Spring Harbor Laboratory, Suffolk, NY, USA) up to 12 June 2020. We looked for observational studies providing any association between COPD and/or asthma and mortality in hospitalized patients with COVID-19. Two investigators (FRV and MH) independently searched for eligible studies in all databases with manual review of all data points. In cases where there was a disagreement regarding the eligibility of a study, a third investigator (LP) was involved for consensus to be reached.

The search algorithm used to perform our search in Medline was: "COVID-19 AND (mortality OR death OR outcome OR risk OR prognostic OR prognosis) AND (retrospective OR prospective OR cross-sectional OR observational OR original)." Similar algorithms were used in the other two databases.

The inclusion criteria were: (i) studies with adult patients hospitalized for COVID-19 and (ii) studies that provided association between COPD and/or asthma and mortality by means of frequencies, odds ratio (OR), or relative risk (RR). There was no language restriction in terms of paper selection. Exclusion criteria were: (i) duplicated or overlapping patient populations and (ii) studies that with less than forty patients. For duplicate or overlapping populations, studies with the larger sample size were included.

\subsection{Data Extraction and Outcomes}

Two independent investigators (F.M.R. and M.H.-M.), who were blinded to each other, extracted and recorded in pre-defined sheet form all relevant information from the eligible studies. The following data were extracted: author, study area and design, hospital location, follow-up period, SARS-CoV-2 diagnostic method, sample size, age, sex, race/ethnicity, baseline comorbidities: coronary artery disease (CAD), heart failure, chronic kidney disease (CKD), dyslipidemia, cerebrovascular accident (CVA) or stroke, diabetes, obesity, chronic obstructive pulmonary disease (COPD), asthma, smoking history, and malignancy history. The primary outcome was in-hospital mortality. The secondary outcomes were need for intubation; admission to an intensive care unit (ICU); and mortality of patients admitted to ICU. 


\subsection{Risk of Bias Assessment}

Risk of bias was assessed by two independent reviewers (F.M.R. and M.H.-M.) using the Quality in Prognosis Studies (QUIPS) tool [20]. Studies were assessed as having low, moderate, serious, or critical risk of bias for the following domains: study participation, study attrition, prognostic factor measurement, confounding measurement and account, outcome measurement, analysis, and reporting.

\subsection{Statistical Analysis}

We estimated the odds ratios (ORs) and their respective 95\% confidence intervals (CI) for all the individual studies. A meta-analysis using the random effects model was performed using the DerSimonian and Laird method [21]. I-squared test was used to assess for heterogeneity for each outcome among trials. Values $<25 \%$ indicated low, $25 \%$ to $70 \%$ moderate, and $>70 \%$ high heterogeneity [21]. Publication bias was reviewed with The Egger test and funnel plots. Sensitivity analyses were performed based on the location of the studies. Sensitivity analysis was also performed for the studies that included only general patient population and the studies that provided adjusted effect size estimates. Meta-regression analyses were performed for important covariates and the outcome of mortality to address high heterogeneity among included studies [21]. We defined a statistical significance level of 5\% with 95\% Confidence Interval. All Statistical analysis was performed with Stata 14.1 (Stata Corp., College Station, TX, USA).

\section{Results}

\subsection{Search Results}

A total of 1595 studies were screened; only thirty observational studies met the final inclusion criteria in the analysis (Figure 1; Table A1) [22-51]. Main study characteristics of COPD and asthma analysis cohorts are abridged in Table 1. Globally, low risk of bias was found (Figure 2). Nine studies were conducted in Asia, seven in the United States, one in Latin America and thirteen in Europe.

\subsection{Patient Characteristics and Primary Events}

A total of 21,309 patients were included; 1465 with COPD and 633 with asthma (Table 2). The mean and/or median age was above 60 years in seventeen studies. In only six studies women accounted for more than $50 \%$ of the population. Studies reporting results in event rates found, overall: frequency of death events in patient with COPD was $34.6 \%(312 / 903)$ compared to $18.4 \%(2991 / 16,240)$ in the non-COPD group; overall frequency of death events in patients with history of asthma was 20.7\% (68/329) compared to $27.2 \%(2253 / 8293)$ in non-asthma group (four out of 23 studies for COPD and two out of seven for asthma studies did not provide the number of death events in the groups of interest; instead, effect size estimates were provided).

\subsection{COPD vs. Non-COPD}

23 studies $(n=19,147)$ were included in the analysis for the outcome of in-hospital mortality. We found that hospitalized COVID-19 patients with COPD had higher risk of death compared to those without COPD but with moderate overall heterogeneity (OR: 2.29; 95\% CI: 1.79-2.93; I² 59.6\%; Figure 3). 


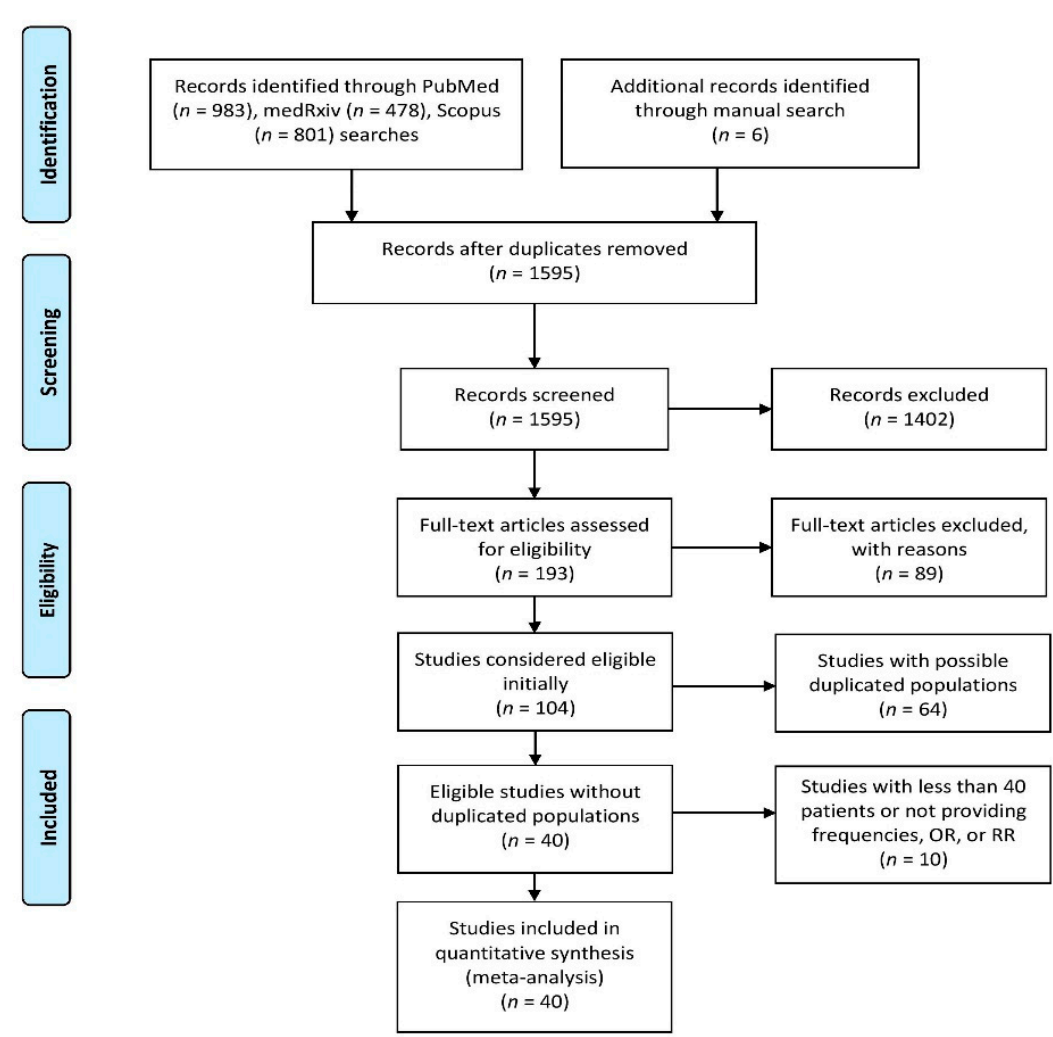

From: Moher D. Liberati A. Tetzlaff J, Altman DG. The PRISMA Group (2009). Preferred Reporting ftems for Systematic Reviews and Meta-

For more information, visit www. prisma-statement.org.

Figure 1. PRISMA Flow Diagram.

Table 1. Characteristics of the included studies. COPD and Asthma analysis cohorts.

\begin{tabular}{|c|c|c|c|c|c|c|c|}
\hline Study & Location & Design & $\begin{array}{c}\text { Enrollment } \\
\text { Start }\end{array}$ & $\begin{array}{l}\text { Enrollment } \\
\text { End }^{\#}\end{array}$ & COPD & Asthma & Sample Size \\
\hline Auld [47] & USA & Retrospective & 6 March & 17 April & $\mathrm{Y}$ & $\mathrm{Y}$ & 217 \\
\hline Borobia [48] & Spain & Retrospective & 25 February & 5 April & $\mathrm{Y}$ & Y & 2226 \\
\hline Cariou [49] & France & Retrospective & 10 March & 31 March & $\mathrm{Y}$ & $\mathrm{N}$ & 1317 \\
\hline Covino [22] & Italy & Retrospective & 1 March & 31 March & $\mathrm{Y}$ & $\mathrm{N}$ & 69 \\
\hline Foy [38] & USA & Retrospective & 4 March & 28 April & $\mathrm{Y}$ & $\mathrm{N}$ & 1198 \\
\hline Gao [43] & China & Retrospective & 1 March & 13 March & $\mathrm{Y}$ & $\mathrm{N}$ & 2877 \\
\hline Huang [44] & China & Retrospective & 25 January & 24 March & $\mathrm{Y}$ & $\mathrm{N}$ & 299 \\
\hline Imam [31] & USA & Retrospective & 1 March & 17 April & $\mathrm{Y}$ & $\mathrm{Y}$ & 1305 \\
\hline Javanian [41] & Iran & Retrospective & 25 February & 12 March & Y & $\mathrm{N}$ & 100 \\
\hline Lee [29] & UK & Prospective & 18 March & 26 April & $\mathrm{Y}$ & $\mathrm{N}$ & 800 \\
\hline Li [39] & China & Retrospective & 10 January & 22 February & $\mathrm{Y}$ & $\mathrm{N}$ & 93 \\
\hline Liu [40] & China & Retrospective & 1 January & 29 February & $\mathrm{Y}$ & $\mathrm{N}$ & 245 \\
\hline Murillo-Zamora [50] & Mexico & Retrospective & 4 March & 5 May & $\mathrm{Y}$ & $\mathrm{Y}$ & 5393 \\
\hline Nowak [32] & Poland & Retrospective & 16 March & 7 April & $\mathrm{Y}$ & $\mathrm{N}$ & 169 \\
\hline Okoh [33] & USA & Retrospective & 10 March & 10 April & $\mathrm{Y}$ & $\mathrm{N}$ & 251 \\
\hline
\end{tabular}


Table 1. Cont.

\begin{tabular}{|c|c|c|c|c|c|c|c|}
\hline Study & Location & Design & $\begin{array}{c}\text { Enrollment } \\
\text { Start }\end{array}$ & $\begin{array}{c}\text { Enrollment } \\
\text { End }^{\#}\end{array}$ & COPD & Asthma & Sample Size \\
\hline Palaiodimos [34] & USA & Retrospective & 9 March & 22 March & $\mathrm{Y}$ & $\mathrm{Y}$ & 200 \\
\hline Russo [36] & Italy & Prospective & February & April & $\mathrm{Y}$ & $\mathrm{N}$ & 192 \\
\hline Satici [28] & Turkey & Retrospective & 2 April & 1 May & $\mathrm{Y}$ & $\mathrm{Y}$ & 681 \\
\hline Shahriarirad [26] & Iran & Retrospective & 20 February & 20 March & $\mathrm{Y}$ & $\mathrm{Y}$ & 113 \\
\hline Tambe [25] & India & Cross-sectional & 31 March & 24 April & $\mathrm{Y}$ & $\mathrm{N}$ & 197 \\
\hline Tanoira [37] & Spain & Retrospective & 3 March & 16 March & $\mathrm{Y}$ & $\mathrm{N}$ & 311 \\
\hline $\mathrm{Xu}[46]$ & China & Retrospective & 10 January & 13 March & $\mathrm{Y}$ & $\mathrm{N}$ & 703 \\
\hline Zhou [42] & China & Retrospective & 19 December & 31 January & $\mathrm{Y}$ & $\mathrm{N}$ & 191 \\
\hline Argenziano * [30] & USA & Retrospective & 1 March & 5 April & Y & $\mathrm{Y}$ & 850 \\
\hline De la Rica [23] & Spain & Retrospective & 15 March & 31 March & $\mathrm{Y}$ & $\mathrm{N}$ & 48 \\
\hline Israelsen [51] & Denmark & Retrospective & 10 March & 23 April & $\mathrm{Y}$ & Y & 175 \\
\hline Rentsch [35] & USA & Retrospective & 8 February & 30 March & $\mathrm{Y}$ & $\mathrm{N}$ & 585 \\
\hline Urra [45] & Spain & Retrospective & $1 \mathrm{March}$ & 15 April & $\mathrm{Y}$ & $\mathrm{N}$ & 172 \\
\hline Di Bella [24] & Italy & Prospective & 25 March & 7 April & $\mathrm{Y}$ & $\mathrm{N}$ & 132 \\
\hline Regina [27] & Switzerland & Retrospective & 1 March & 25 March & Y & $\mathrm{N}$ & 200 \\
\hline
\end{tabular}

Notes: * only the subset of hospitalized patients was included for analysis. ${ }^{\#}$ Year 2020. Abbreviations: USA = United States of America, $\mathrm{UK}=$ United Kingdom, COPD = chronic obstructive pulmonary disease, $\mathrm{Y}=\mathrm{Yes}, \mathrm{N}=\mathrm{No}$.

\begin{tabular}{|c|c|c|c|c|c|c|c|c|c|c|c|c|c|c|c|}
\hline & & \multirow{2}{*}{\multicolumn{3}{|c|}{ High }} & & & & & & & & & & & \\
\hline & - & & & & & & & & & & & & & & \\
\hline & $\theta$ & \multicolumn{3}{|c|}{ Moderate } & & & & & & & & & & & \\
\hline & 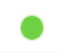 & \multicolumn{3}{|c|}{ Low } & & & & & & & & & & & \\
\hline & $\bullet$ & \multicolumn{3}{|c|}{ Not applicable } & & & & & & & & & & & \\
\hline & 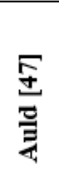 & 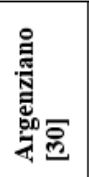 & 獣 & 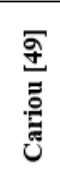 & 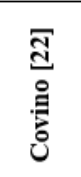 & 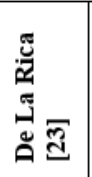 & 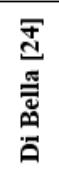 & 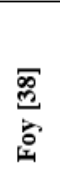 & $\begin{array}{l}\overline{9} \\
\frac{9}{8} \\
\stackrel{\mathscr{E}}{0}\end{array}$ & 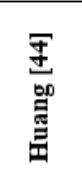 & 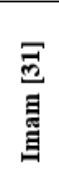 & 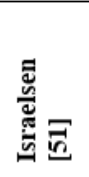 & 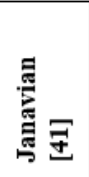 & ฐ্ডે & $\begin{array}{l}\overline{2} \\
\overline{3}\end{array}$ \\
\hline \multirow{2}{*}{$\begin{array}{l}\text { 1.Study } \\
\text { participation } \\
\text { 2. Study attrition }\end{array}$} & ○ & 0 & - & O & - & 0 & 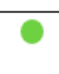 & 0 & 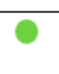 & 0 & 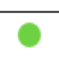 & 0 & - & 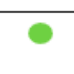 & $\odot$ \\
\hline & $\bullet$ & $\bullet$ & $\bullet$ & 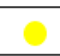 & - & . & $\bullet$ & $\bullet$ & $\bullet$ & • & $\bullet$ & $\bullet$ & • & - & $\bullet$ \\
\hline \multirow{3}{*}{$\begin{array}{l}\text { 3. Prognostic factor } \\
\text { measurement } \\
\text { 4. Outcome } \\
\text { measurement } \\
5 \text {. Study cofounding }\end{array}$} & $\bullet$ & ○ & 0 & - & - & ○ & $\bullet$ & 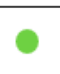 & 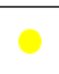 & - & $\bullet$ & 0 & 0 & $\theta$ & 0 \\
\hline & 0 & . & $\theta$ & 0 & $\bullet$ & 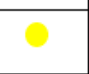 & - & 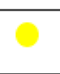 & 0 & $\bullet$ & 0 & 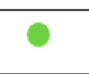 & $\bullet$ & ○ & $\gamma$ \\
\hline & $\bullet$ & - & 앙 & - & - & $\bullet$ & 8 & 8 & 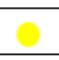 & 0 & 0 & 을 & 단 & 8 & 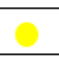 \\
\hline \multirow[t]{2}{*}{$\begin{array}{l}6 . \text { Statistical } \\
\text { analysis and } \\
\text { reporting }\end{array}$} & - & - & - & - & - & $\bullet$ & - & - & - & 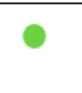 & - & - & $\bullet$ & - & - \\
\hline & 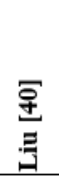 & 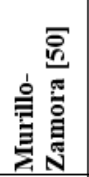 & 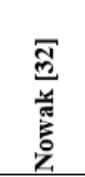 & $\begin{array}{l}\overline{0} \\
\frac{0}{0} \\
\overline{0}\end{array}$ & 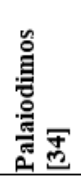 & 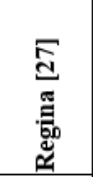 & 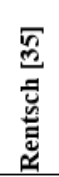 & 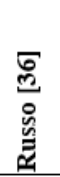 & 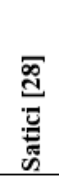 & 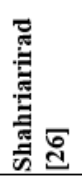 & 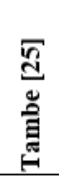 & 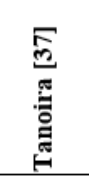 & $\frac{\bar{m}}{\stackrel{5}{+}}$ & 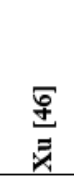 & $\begin{array}{l}\overline{\mathrm{g}} \\
\frac{\mathrm{g}}{\mathrm{g}} \\
\end{array}$ \\
\hline \multirow{2}{*}{$\begin{array}{l}\text { 1.Study } \\
\text { participation } \\
\text { 2. Study attrition }\end{array}$} & 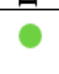 & 0 & 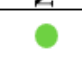 & 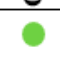 & 0 & 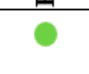 & 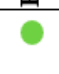 & 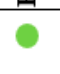 & 0 & 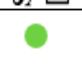 & 0 & $\theta$ & 0 & 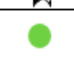 & 0 \\
\hline & - & - & • & 0 & - & - & - & • & • & $\bullet$ & - & - & $\bullet$ & $\bullet$ & 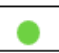 \\
\hline \multirow{3}{*}{$\begin{array}{l}\text { 3. Prognostic factor } \\
\text { measurement } \\
\text { 4. Outcome } \\
\text { measurement } \\
\text { 5. Study cofounding }\end{array}$} & $\theta$ & - & 0 & 0 & - & 0 & 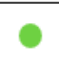 & 0 & 0 & $\theta$ & $\theta$ & . & - & ○ & 0 \\
\hline & $\bullet$ & 0 & 0 & 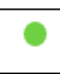 & $\bullet$ & $\bullet$ & 0 & 0 & 0 & 0 & 0 & - & - & ○ & 0 \\
\hline & 잉 & 0 & 당 & 0 & 당 & 0 & 0 & $\theta$ & $\theta$ & 0 & 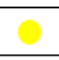 & 잉 & 앙 & 0 & 0 \\
\hline
\end{tabular}

Figure 2. Quality in Prognosis Studies-QUIPS Tool: Risk of Bias Assessment. 
Table 2. Baseline characteristics of patients per included studies. Described in absolute and relative frequencies.

\begin{tabular}{|c|c|c|c|c|c|c|c|c|c|c|c|c|}
\hline Study & Age & $\begin{array}{c}\text { Female } \\
n(\%)\end{array}$ & $\begin{array}{c}\text { Diabetes } \\
n(\%)\end{array}$ & $\begin{array}{c}\text { Hypertension } \\
n(\%)\end{array}$ & $\begin{array}{l}\text { CAD } \\
n(\%)\end{array}$ & $\begin{array}{c}\text { Heart Failure } \\
n(\%)\end{array}$ & $\begin{array}{l}\text { CKD } \\
n(\%)\end{array}$ & $\begin{array}{l}\text { CVA } \\
n(\%)\end{array}$ & $\begin{array}{c}\text { Smoking } \\
n(\%)\end{array}$ & $\begin{array}{c}\text { Malignancy } \\
n(\%)\end{array}$ & $\begin{array}{l}\text { COPD } \\
n(\%)\end{array}$ & $\begin{array}{c}\text { Asthma } \\
n(\%)\end{array}$ \\
\hline Auld [47] & $64(54-73)^{\mathrm{b}}$ & $98(45.2)$ & 99 (45.4) & $134(61.7)$ & $31(14.3)$ & $41(18.9)$ & $58(26.7)$ & NA & NA & NA & $21(9.7)$ & $19(8.8)$ \\
\hline Borobia [48] & $61(46-78)^{\mathrm{b}}$ & $1152(51.8)$ & 381 (17.1) & $920(41.3)$ & NA & NA & $174(7.8)$ & NA & $157(7.1)$ & $133(6.0)$ & $153(6.9)$ & $115(5.2)$ \\
\hline Cariou [49] & $69.8(13)^{\mathrm{a}}$ & $462(35.1)$ & NA & $1003(76.1)$ & $336(25.5)$ & 140 (10.6) & $60(4.5)$ & $163(12.4)$ & 447 (33.9) & NA & $133(10.1)$ & NA \\
\hline Covino [22] & $84(82-89)^{\mathrm{d}}$ & $32(46.4)$ & $9(13.0)$ & $41(59.4)$ & $21(30.4)$ & $21(30.4)$ & NA & $20(29.0 \%)$ & NA & $3(4.3)$ & $7(10.1)$ & NA \\
\hline Foy [38] & NA & 534 (44.8) & NA & NA & NA & NA & NA & NA & NA & NA & $273(22.8)$ & NA \\
\hline Gao [43] & NA & NA & 387 (13.6) & $850(29.5)$ & $295(10.25)$ & $23(0.8)$ & $29(1.0)$ & $52(1.8)$ & $190(6.6)$ & $49(1.7)$ & $31(1.1)$ & $\mathrm{NA}$ \\
\hline Huang [44] & $53.4(16.7)^{\mathrm{a}}$ & $139(46.5)$ & 35 (11.7) & $74(24.7)$ & $18(6)$ & NA & NA & $13(4.3)$ & $48(16.1)$ & $9(3.0)$ & $8(2.6)$ & NA \\
\hline Imam [31] & $61.0(16.3)^{\mathrm{a}}$ & $603(46.2)$ & $393(30.1)$ & $734(56.2)$ & $208(15.9)$ & $75(5.7)$ & $228(17.5)$ & $95(7.3)$ & $356(27.3)$ & $83(6.4)$ & $107(8.2)$ & $115(8.8)$ \\
\hline Javanian [41] & $60.12(13.87)^{\mathrm{b}}$ & $49(49)$ & $34(34.0)$ & $31(31.0)$ & $22(22.0)$ & NA & 11 (11.0) & $3(3.0)$ & NA & $4(4.0)$ & $12(12.0)$ & NA \\
\hline Lee [29] & $69(59.0-76.0)^{\mathrm{b}}$ & 349 (43.6) & 131 (16.4) & $247(30.9)$ & NA & NA & NA & NA & NA & $800(100)$ & $61(7.6)$ & NA \\
\hline Liu [40] & $53.95(16.9)^{a}$ & 131 (53.5) & $23(9.4)$ & $52(21.2)$ & $18(7.3)$ & NA & NA & NA & $10(4.01)$ & $9(3.7 \%)$ & $8(3.3)$ & NA \\
\hline $\begin{array}{l}\text { Murillo- } \\
\text { Zamora } \\
\text { [50] }\end{array}$ & NA & 1,961 (36.4) & 1,677 (31.1) & 1,973 (36.6) & NA & NA & $299(5.5)$ & NA & NA & NA & $273(5.1)$ & $146(2.7)$ \\
\hline Nowak [32] & $63.7(19.6)^{\mathrm{a}}$ & $82(48.5)$ & $32(18.9)$ & $80(47.3)$ & $52(30.8)$ & NA & $35(20.7)$ & $58(34.3)$ & NA & $35(20.7)$ & $22(13.3)$ & NA \\
\hline Okoh [33] & $62(49-74)^{\mathrm{b}}$ & $122(48.6)$ & $115(45.8)$ & $175(69.7)$ & 49 (19.5) & $50(19.9)$ & 46 (18.3) & $28(11.1)$ & NA & $22(8.7)$ & $23(9.2)$ & NA \\
\hline $\begin{array}{l}\text { Palaiodimos } \\
\text { [34] }\end{array}$ & $64(50.0-73.5)^{\mathrm{b}}$ & $102(51.0)$ & 79 (39.5) & $152(76.0)$ & $33(16.5)$ & $34(17.0)$ & 41 (20.5) & $22(11.0)$ & NA & $11(5.5)$ & $28(14.0)$ & $27(13.5)$ \\
\hline $\begin{array}{l}\text { Russo [36] } \\
\text { Satici [28] }\end{array}$ & $\begin{array}{l}67.7(15.2)^{\mathrm{a}} \\
56.9(15.7)^{\mathrm{a}}\end{array}$ & $\begin{array}{c}77(40.1) \\
334(49.0)\end{array}$ & $\begin{array}{c}42(21.9) \\
191(28.0)\end{array}$ & $\begin{array}{l}111(57.8) \\
234(34.4)\end{array}$ & $\begin{array}{l}26(13.5) \\
62(9.1)\end{array}$ & $\begin{array}{l}20(10.4) \\
19(2.8)\end{array}$ & $\begin{array}{l}12(6.3) \\
24(3.5)\end{array}$ & $\begin{array}{c}16(8.3) \\
\text { NA }\end{array}$ & $\begin{array}{c}16(8 . .0) \\
\text { NA }\end{array}$ & $\begin{array}{l}\mathrm{NA} \\
9(1.3)\end{array}$ & $\begin{array}{l}26(13.5) \\
28(4.1)\end{array}$ & $\begin{array}{c}\mathrm{NA} \\
43(6.3)\end{array}$ \\
\hline $\begin{array}{c}\text { Shahriarirad } \\
{[26]}\end{array}$ & $53.75(16.58)^{a}$ & $42(37.2)$ & $16(14.2)$ & $22(19.5)$ & $16(14.2)$ & NA & $6(5.3)$ & NA & NA & $1(0.9)$ & $9(7.9)$ & $7(6.2)$ \\
\hline $\begin{array}{l}\text { Tambe [25] } \\
\text { Tanoira [37] }\end{array}$ & $\begin{array}{c}45.8(17.3)^{\mathrm{a}} \\
\text { NA }\end{array}$ & $\begin{array}{c}107 \text { (54.5) } \\
\text { NA }\end{array}$ & $\begin{array}{c}42(21.3) \\
\text { NA }\end{array}$ & $\begin{array}{c}60 \text { (30.5) } \\
\text { NA }\end{array}$ & $\begin{array}{l}4(2.0) \\
\mathrm{NA}\end{array}$ & $\begin{array}{l}\text { NA } \\
\text { NA }\end{array}$ & $\begin{array}{c}2(1.0) \\
\mathrm{NA}\end{array}$ & $\begin{array}{l}\text { NA } \\
\text { NA }\end{array}$ & $\begin{array}{l}\text { NA } \\
\text { NA }\end{array}$ & $\begin{array}{l}\text { NA } \\
\text { NA }\end{array}$ & $\begin{array}{c}10(5.1) \\
\mathrm{NA}\end{array}$ & $\begin{array}{l}\text { NA } \\
\text { NA }\end{array}$ \\
\hline Zhou [42] & $56 \cdot 0(46-67)^{\mathrm{b}}$ & $72(37.7)$ & $36(18.8)$ & $58(30.4)$ & $15(7.9)$ & NA & $2(1.0)$ & NA & $11(5.8)$ & $2(1.0)$ & $6(3.1)$ & NA \\
\hline $\begin{array}{c}\text { Argenziano } \\
{[30]}\end{array}$ & $63.0 \underset{\mathrm{b}}{(50.0-75.0)}$ & $339(39.9)$ & $333(39.2)$ & $525(61.8 \%)$ & $115(13.5)$ & $91(10.7)$ & $125(14.7)$ & $72(8.5)$ & $198(23.3)$ & $63(7.4)$ & $56(6.6)$ & $88(10.4)$ \\
\hline $\begin{array}{l}\text { De la Rica [23] } \\
\text { Israelsen [51] }\end{array}$ & $\begin{array}{l}65.98(13.9)^{\mathrm{a}} \\
71(55-81)^{\mathrm{b}}\end{array}$ & $\begin{array}{l}16(33.3) \\
90(51,4)\end{array}$ & $\begin{array}{l}11(22.9) \\
46(26.3)\end{array}$ & $\begin{array}{l}33(68.8) \\
73(41.7)\end{array}$ & $\begin{array}{l}14(29.2) \\
90(51.4)\end{array}$ & $\begin{array}{l}\text { NA } \\
\text { NA }\end{array}$ & $\begin{array}{l}8(16.7) \\
\text { NA }\end{array}$ & $\begin{array}{l}\text { NA } \\
\text { NA }\end{array}$ & $\begin{array}{l}10(20.8) \\
63(36.0)\end{array}$ & $\begin{array}{l}10(20.8) \\
\text { NA }\end{array}$ & $\begin{array}{l}5(10.4) \\
11(6.3)\end{array}$ & $\begin{array}{c}\text { NA } \\
20(11.4)\end{array}$ \\
\hline Rentsch [35] & $66.1 \underset{\mathrm{b}}{(60.4-71.0)}$ & $27(4.6)$ & $260(44.4)$ & $423(72.3)$ & $163(27.3)$ & NA & NA & NA & $338(57.8)$ & $83(14.2)$ & $90(15.4)$ & $45(7.7)$ \\
\hline Urra [45] & NA & $68(39.5)$ & $39(22.7)$ & $87(50.6)$ & $28(16.3)$ & NA & NA & NA & NA & NA & $17(9.9)$ & NA \\
\hline Di Bella [24] & $66(55-75.8)^{\mathrm{b}}$ & $42(31.8)$ & $33(25.0)$ & $55(41.7)$ & $24(18.2)$ & NA & NA & NA & $12(9.1)$ & NA & $10(7.6)$ & NA \\
\hline Regina [27] & $70(55-81)^{\mathrm{b}}$ & $80(40.0)$ & $43(21.5)$ & 87 (43.5) & 35 (17.5) & NA & 28 (14.0) & $21(10.5)$ & NA & NA & $16(8.0)$ & $8(4.0)$ \\
\hline
\end{tabular}

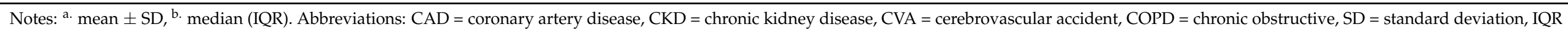
$=$ interquartile range, $\mathrm{NA}=$ non-available. 


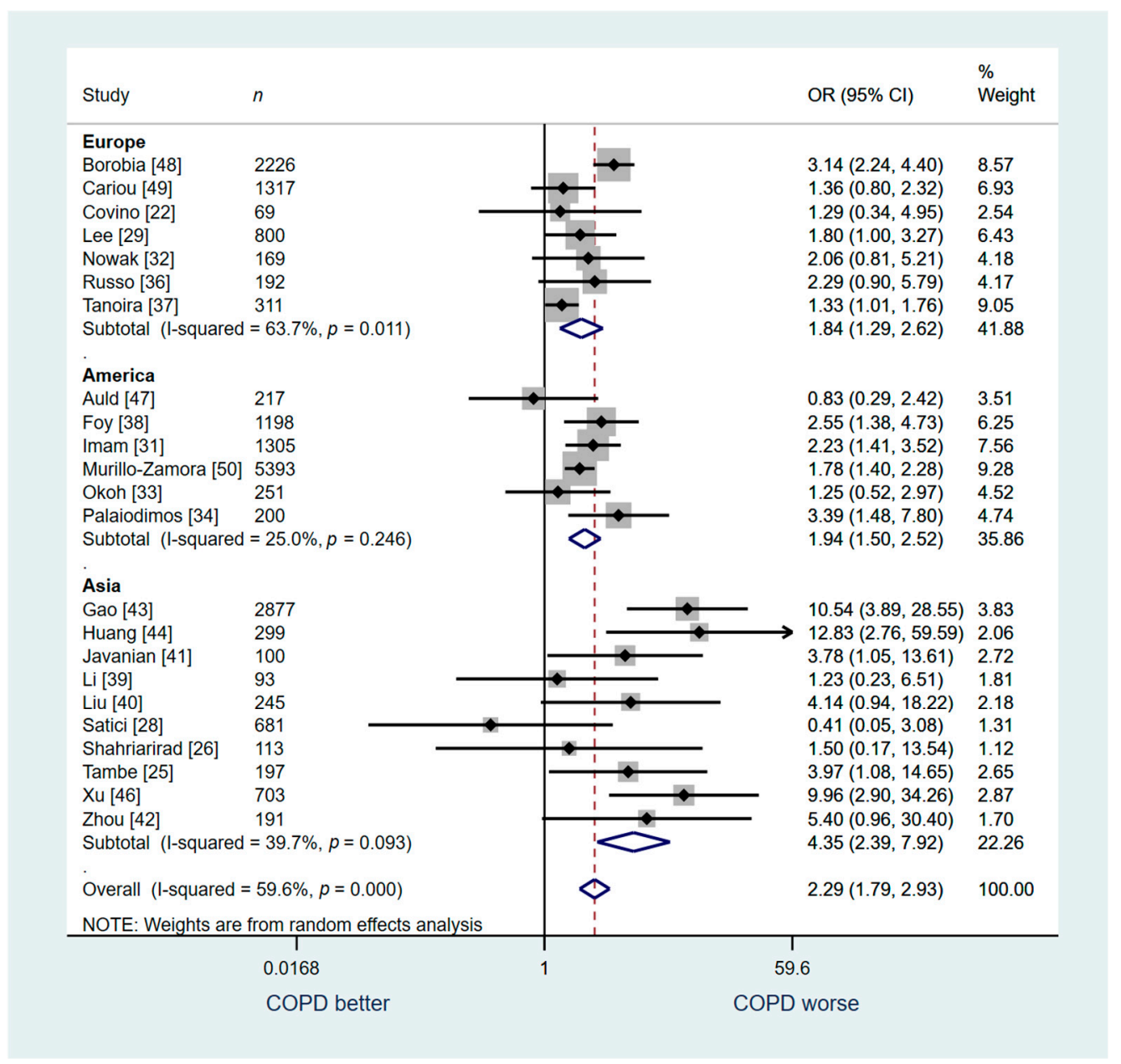

Figure 3. Overall analysis: COPD vs. no COPD for in-hospital mortality.

A sensitivity analysis was conducted for studies from Europe $(n=7)$, America $(n=6)$, and Asia $(n=10)$. Similar to the overall analysis, our sensitivity analysis for studies conducted in Europe revealed a higher risk of death among patients with COPD compared to those without COPD (OR: 1.84; 95\% CI: 1.29-2.62; I2 63.7\%, Figure 3). We found similar association for the studies from in America with low heterogeneity (OR: 1.94; 95\% CI: 1.50-2.52; $\mathrm{I}^{2} 25.0 \%$, Figure 3) and among studies conducted in Asia (OR: 4.35; 95\% CI: 2.39-7.62; $\mathrm{I}^{2} 39.7 \%$, Figure 3).

Another sensitivity analysis was conducted for studies that included general patient population (Studies: 18, $n=16,493$ ). Five studies that included a pre-specified special population (e.g., only critically ill patients, only diabetics, only patients with cancer, only a specific age or racial group) were excluded for this analysis. This analysis demonstrated a similar association between COPD and increased risk for in-hospital death (OR: 2.74; 95\% CI: 2.04-3.68; I² 64.1\%, Figure 4).

Only one study that reported results on the secondary outcome of ICU mortality was included, therefore meta-analysis was not relevant.

Six studies $(n=4056)$ were included for secondary outcome analysis of admission to an ICU. COPD patients were not associated with a higher risk of admission to ICU compared to patients without COPD, but a trend was noted (OR: 1.39; 95\% CI: 0.79-2.44; $I^{2} 57.5 \%$; Figure 5). 


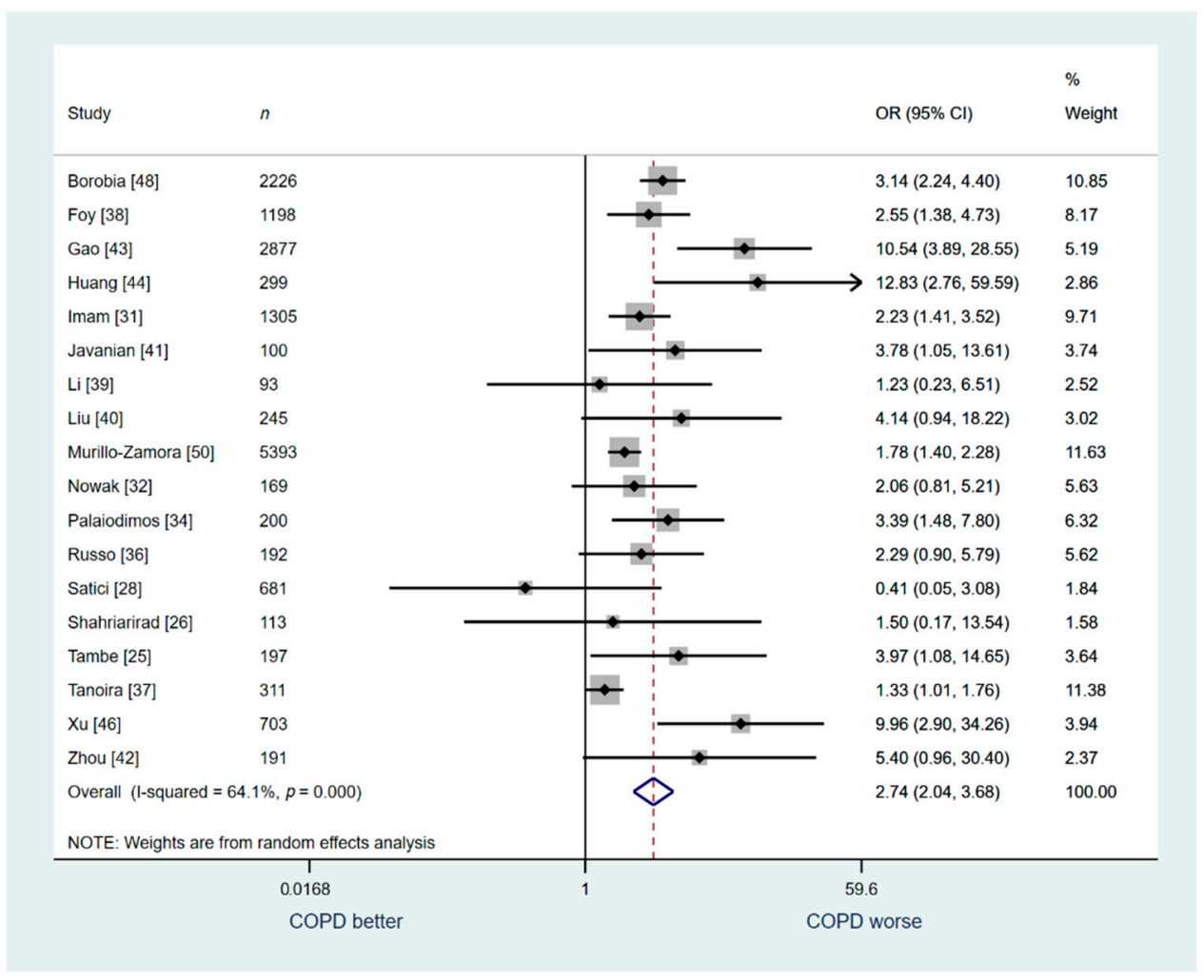

Figure 4. Analysis of the studies that included only general patient population: COPD vs. no COPD for in-hospital mortality.

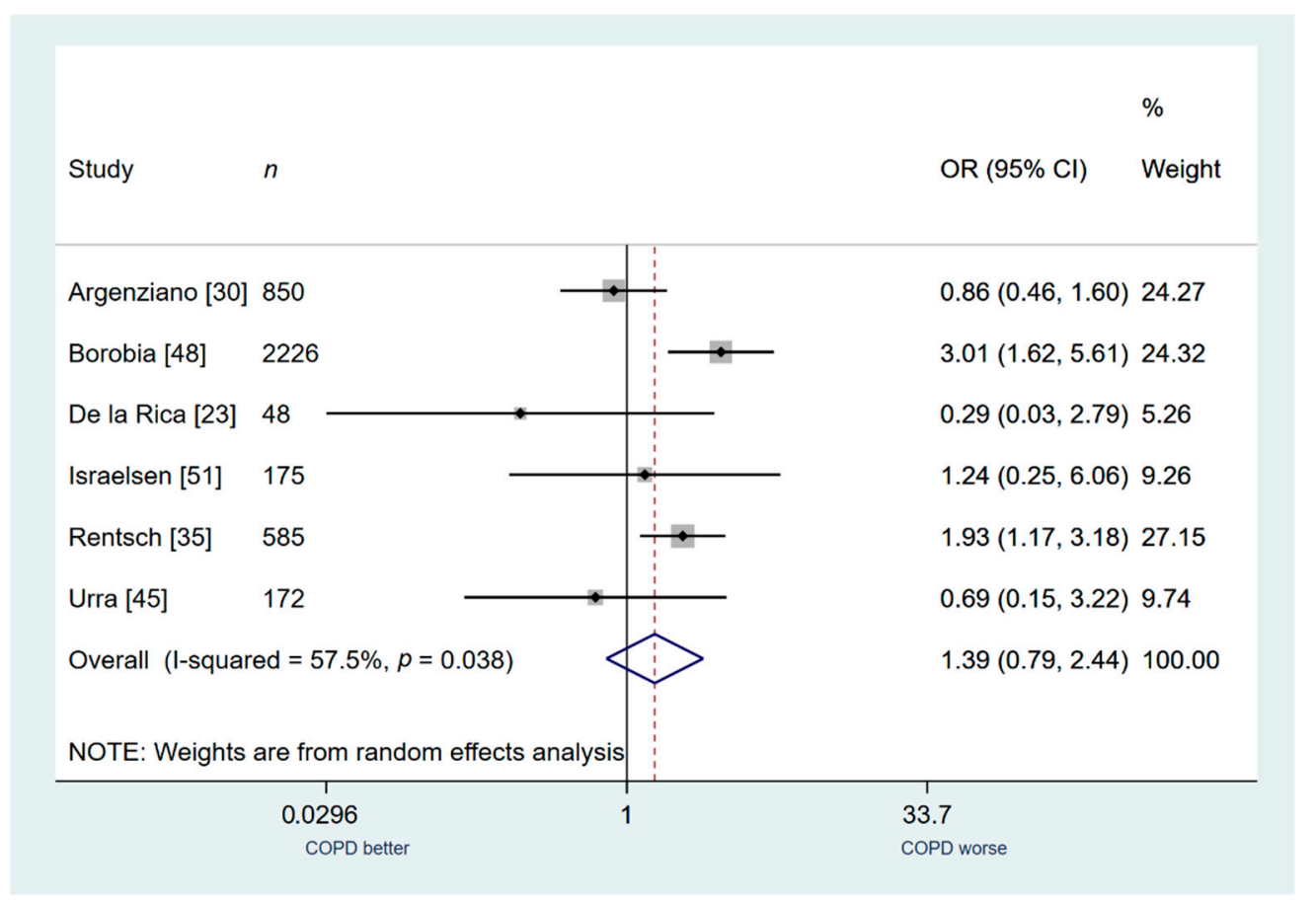

Figure 5. COPD vs. no COPD for the outcome of admission in the ICU.

Finally, only three studies $(n=532)$ were included in secondary outcome analysis regarding Intubation. Patients with COPD were found to be associated with a higher risk of intubation compared to patients without COPD (OR: 2.03; 95\% CI: $1.09-3.80 ; I^{2} 0.00 \%$; Figure 6). 


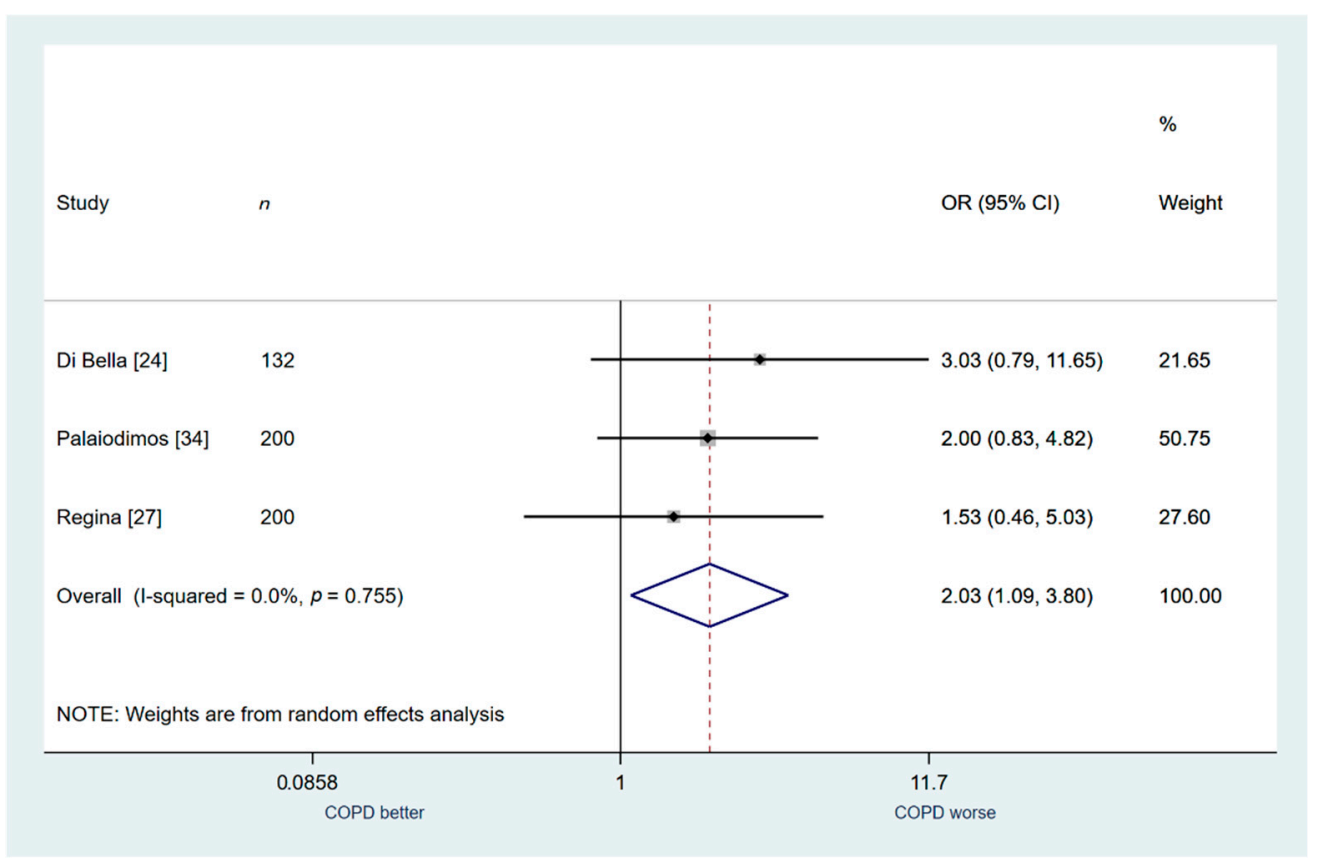

Figure 6. COPD vs. no COPD for the outcome of intubation.

\subsection{Asthma vs. Non-Asthma}

Seven studies $(n=10,136)$ were included in the secondary outcomes analysis for In-hospital mortality. Asthma patients were not associated with a higher risk of death compared to patients without asthma; the heterogeneity was low (OR: 0.87; 95\% CI: $0.68-1.10 ; I^{2} 0.0 \%$; Figure 7).

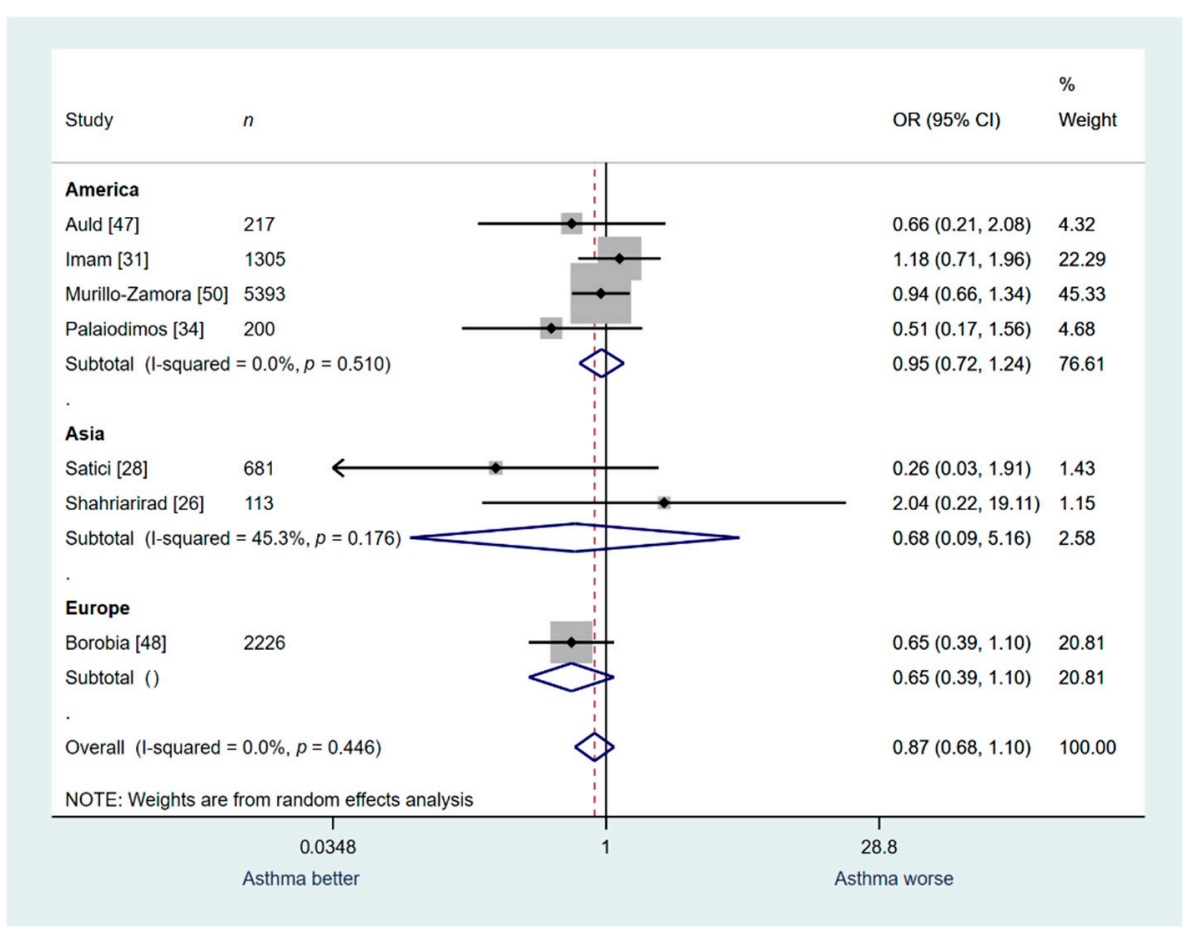

Figure 7. Overall analysis: asthma vs. no asthma for in-hospital mortality.

A sensitivity analysis was conducted for studies that were performed in Europe $(n=1)$, America $(n=4)$, and Asia $(n=2)$. Similarly, to the overall analysis, the sensitivity analysis 
based on the continent that the study was conducted revealed no significant association between asthma and in-hospital mortality (Figure 7).

Another sensitivity analysis was conducted for studies that included general patient population (Studies: 6, $n=9918$ ). One study that included a pre-specified special population (only critically ill patients) was excluded for this analysis. Similarly, this analysis demonstrated no association between asthma and risk for in-hospital death (OR: 0.87; 95\% CI: $0.66-1.14 ; I^{2} 0.0 \%$, Figure 8).

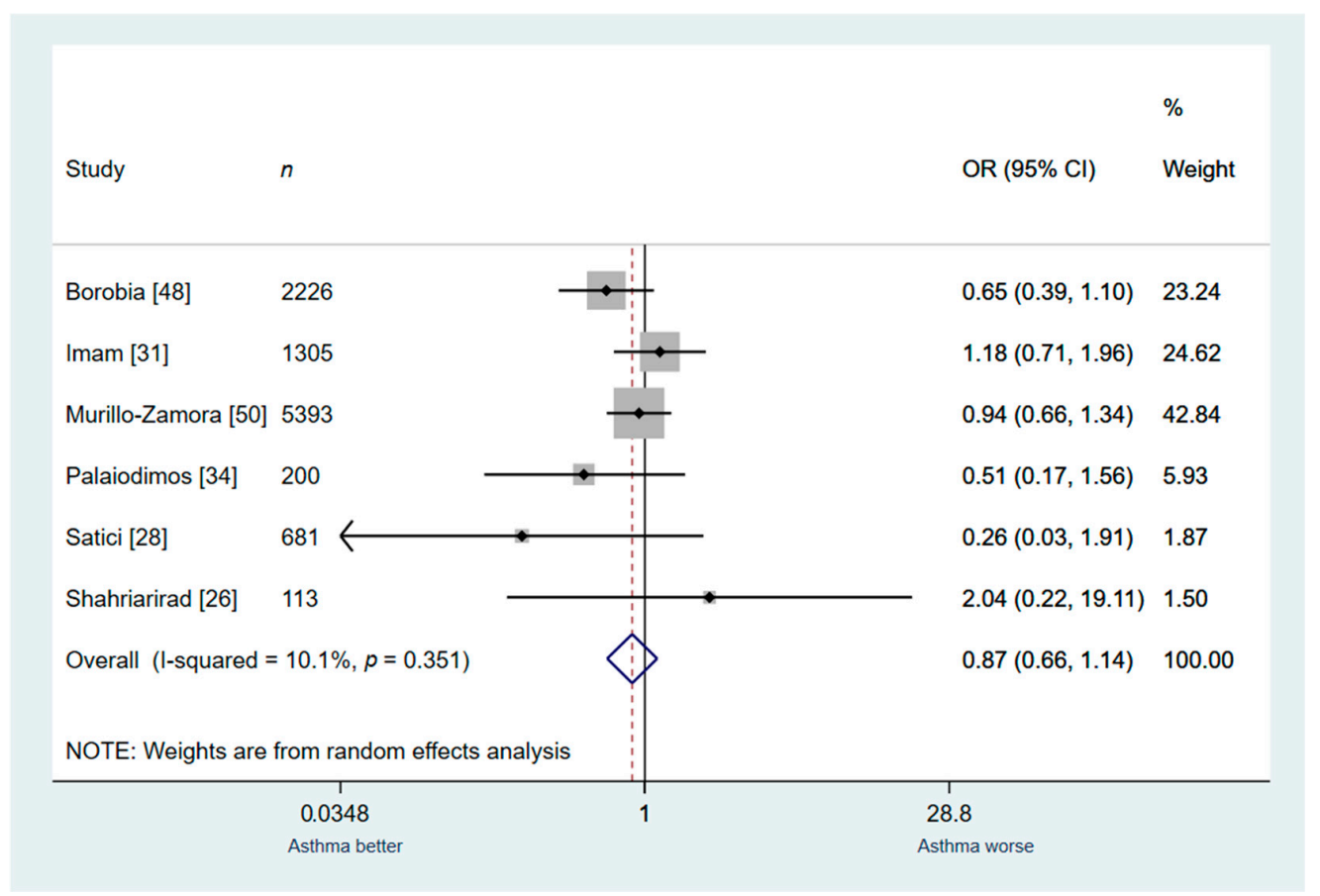

Figure 8. Analysis of the studies that included only general patient population: asthma vs. no asthma for in-hospital mortality.

\subsection{Quality Assessment and Heterogeneity}

Eggers's test and funnel plots were used to assess for publication bias. Visual assessment of the funnel plot was not suggestive for publication bias (Figure A1). Egger test for potential publication bias in a meta-analysis via funnel plot asymmetry was not indicative of publication bias $(p=0.388)$.

Meta-regression analysis was performed based on several major covariates (age: $p=0.838$, female sex: $p=0.330$, hypertension: $p=0.743$, coronary artery disease: $p=0.387$, heart failure: $p=0.568$, chronic kidney disease: $p=0.979$, diabetes: $p=0.924$ and malignancy history: $p=0.688$ ). No significant interactions were found between the covariates and the outcome of mortality. The results of the meta-regression are found in Table 3. 
Table 3. Results of the meta-regression analyses.

\begin{tabular}{|c|c|c|c|c|c|c|}
\hline \multirow[b]{2}{*}{ Variables } & \multicolumn{3}{|c|}{ COPD } & \multicolumn{3}{|c|}{ Asthma } \\
\hline & Coefficient & Standard Error & $p$-Value & Coefficient & Standard Error & $p$-Value \\
\hline Age & -0.009 & 0.006 & 0.146 & 0.040 & 0.175 & 0.835 \\
\hline Female & 0.031 & 0.013 & 0.037 & -0.026 & 0.024 & 0.330 \\
\hline HTN & -0.012 & 0.008 & 0.114 & -0.004 & 0.013 & 0.743 \\
\hline CAD & -0.006 & 0.014 & 0.648 & 0.149 & 0.148 & 0.387 \\
\hline $\mathrm{HF}$ & -0.016 & 0.027 & 0.584 & -0.033 & 0.049 & 0.568 \\
\hline CKD & -0.015 & 0.022 & 0.489 & 0.001 & 0.023 & 0.979 \\
\hline Diabetes & -0.026 & 0.011 & 0.023 & 0.002 & 0.019 & 0.924 \\
\hline Malignacy & -0.005 & 0.005 & 0.316 & 0.077 & 0.173 & 0.688 \\
\hline CVA & -0.019 & 0.021 & 0.371 & & & \\
\hline Smoking & -0.026 & 0.013 & 0.108 & & & \\
\hline
\end{tabular}

Abbreviations: $\mathrm{HTN}=$ hypertension, $\mathrm{CAD}=$ coronary artery disease, $\mathrm{HF}=$ heart failure, $\mathrm{CKD}=$ chronic kidney disease, $\mathrm{CVA}=$ cerebrovascular accident. Note: Meta-regression is a meta-analytic tool to examine the impact of moderator variables on study effect size. It cannot be used to evaluate the association of moderator variables with the outcome of interest.

\section{Discussion}

Our study was a systematic review and meta-analysis of observational studies exploring the association between COPD and asthma with mortality in adult hospitalized patients with COVID-19. The findings of our study are: (i) overall, death was more likely to occur in inpatients with COPD compared with patients without COPD (moderate heterogeneity); (ii) no significant association between history of asthma and in-hospital mortality was revealed.

\subsection{COPD and Mortality in Patients with COVID-19}

Our findings are consistent with the results of an early review that included a total of 2473 patients from studies conducted only in Asia published until late March 2020 [52]. COPD patients were found to have an $88 \%$ higher risk of severe disease and $60 \%$ higher mortality compared to patients without COPD [52]. Conversely, other larger observational studies have revealed no association of COPD with worse outcomes. Petrilli et al. reported no association between history of COPD and development of critical illness in a cohort of more than 5729 patients from New York [53]. Similarly, a recently published retrospective study of 6916 from California demonstrated no association between history of COPD and in-hospital mortality [54]. Given the heterogeneity of the results across the literature, our meta-analysis attempted to answer this significant question by utilizing a total sample of 19,147 patients across continents.

Seasonal influenza and bacterial pneumonia have been clearly linked to worse outcomes and increased mortality in patients with COPD [55-58]. On the other hand, the reports regarding the impact of COPD in patients with H1N1 Influenza were contradictory [59-64]. Although SARS-CoV and SARS-CoV-2 share 79.6\% of their genome, COPD as a comorbidity was underreported during the SARS epidemic of 2003 caused by SARS-CoV, making the comparison difficult [42,65-74].

The higher mortality noted in patients with COPD in our study could be related to smoking, which is considered to be a potential up-regulator of the Angiotensin Converting Enzyme-2 (ACE2) receptor, the adhesion site for the SARS-CoV-2 virus. This hypothesis has been contested [75-79]. COPD has genotypic and phenotypic findings that makes it the prime disease for SARS-CoV-2 targeting. In 2015, Kim et al. showed genes associated with ACE2 level expression were more likely to be found in COPD patients [80], and this has been confirmed by other research groups even in non-smoking COPD patients $[76,81,82]$. The pathophysiology is presumed to be linked to inflammatory signaling secondary to smoking and to ACE2 expression upregulation in secretory cells expressing ACE2 [82-84]. Another possible explanation into why we found patients with COPD to have higher mortality in COVID19 is because they are general sicker than the general population. More so, patients with COPD have more comorbidities than the general population or patients 
with other chronic diseases [85]; with two to five times higher risk of ischemic heart disease, cardiac dysrhythmia, heart failure, diseases of the pulmonary circulation, and diseases of the arteries [86].

\subsection{Asthma and Mortality in Patients with COVID-19}

Patient with asthma facing an upper respiratory illness would be expected to have a higher risk for a worse outcome when compared to healthy individuals [87]. In the present analysis patients with a history of asthma were not found to have increased inhospital mortality. Several recent large cohort studies of asthma patients confirm our findings [88-91].

Adults with asthma are at increased risk of invasive pneumococcal infections [92,93], Bacterial pneumonias have been associated with asthma exacerbations but not necessarily with increased mortality [94]. Correspondingly, viral respiratory infections have been linked with acute asthma exacerbations $[95,96]$. Seasonal influenza increased the risk of acute asthma exacerbation in elderly patients, but, similarly to our findings, no reports have clearly showed increased mortality [97-100]. Likewise, during the 2009 H1N1 pandemic adult asthmatics were not found to be at higher risk of worse outcomes $[4,55,59,101-103]$.

Asthma has been shown to have decreased ACE2 expression in murine models [104]. Different asthma phenotypes could have different interactions with the COVID-19 virus: patients with low peripheral blood eosinophils have increased ACE2 expression [105], while ACE2 expression is downregulated in patients with chronic history of respiratory allergies by interleukin modulation [106,107]. This decreased expression of ACE2 throughout the body could explain why asthmatic patients had in general better outcomes than patients with COPD.

\subsection{Strengths and Limitations}

The main strengths of our study are its strict methodology, robust analysis, and large number of included studies and overall patient sample size. Three continents and most of the countries that had high COVID-19 incidence at the time of the analysis were represented. Sensitivity and meta-regression analyses were performed. The main limitations of our study are the observational nature of the primary studies and the lack of spirometry data confirming the diagnosis of obstructive lung disease. COPD is often underdiagnosed in the general population [108]. Another important limitation is the lack of data on the severity of COPD and asthma; thus, we could not estimate the associations of well-controlled versus poorly controlled obstructive lung disease with in-hospital mortality. A patient-level metaanalysis would be needed to assess this particularly important parameter. Moreover, our meta-analysis was limited by moderate heterogeneity, which we tried to rectify by using a random-effects model and performing sensitivity and meta-regression analyses. Important to note, different experimental treatment regimens of COVID-19 across continents may have affected general overall mortality as well as health-care access and level of care to these treatments. Finally, several observational studies have been published after we completed this meta-analysis. However, our study included a large number of studies and patients from different parts of the world, and it is unlikely that the inclusion of the additional recent studies would change our results.

\section{Conclusions}

In conclusion, this systematic review and meta-analysis showed that patients with history of COPD who were hospitalized with COVID-19 were associated with higher inhospital mortality. Further prospective studies are needed to assess whether this association is independent or not. In contrast, asthmatic patients with COVID-19 were not found to be associated with higher mortality.

Supplementary Materials: The following are available online at https://www.mdpi.com/article/10 $.3390 /$ jcm10102087/s1, Table S1: PRSIMA Checklist. 
Author Contributions: Conceptualization, F.M.R., L.P. and M.H.-M.; methodology, L.P. and D.K.; software, D.K. and C.G.B.; validation, P.G., G.N., C.G.B., R.E. and M.L.; formal analysis, L.P.; investigation, M.H.-M. and F.M.R.; resources, C.D.S. and G.N.; data curation C.G.B., D.K. and L.P.; writing-original draft preparation, F.M.R., P.G., L.P., C.G.B., R.E. and C.D.S.; writing-review and editing, M.L., G.N. and M.H.-M.; visualization, F.M.R. and D.K.; supervision, L.P.; project administration, M.H.-M.; funding acquisition, L.P. All authors have read and agreed to the published version of the manuscript.

Funding: This research received no external funding.

Institutional Review Board Statement: This meta-analysis is exempt from Institutional Review Board ethics approval because data was collected and synthesized from publicly available clinical studies in which informed consent was already obtained by the study investigators, and the metaanalysis will be addressing very similar questions to the research question for which the data was collected (and to which patients gave consent). The study was conducted according to the guidelines of the Declaration of Helsinki.

Informed Consent Statement: Patient consent was waived due to study being meta-analysis, individual consents were obtained from individual studies by studies principal investigators.

Data Availability Statement: Data is contained within the article or Supplementary Material. Further patient or study level data supporting reported results are available on request; can be sent as excel sheet file or another statistical file.

Acknowledgments: Andrew Wiznia and Jason Leider from Jacobi Medical Center, Albert Einstein College of Medicine for providing continuous support to research endeavors.

Conflicts of Interest: The authors declare no conflict of interest.

\section{Appendix A}
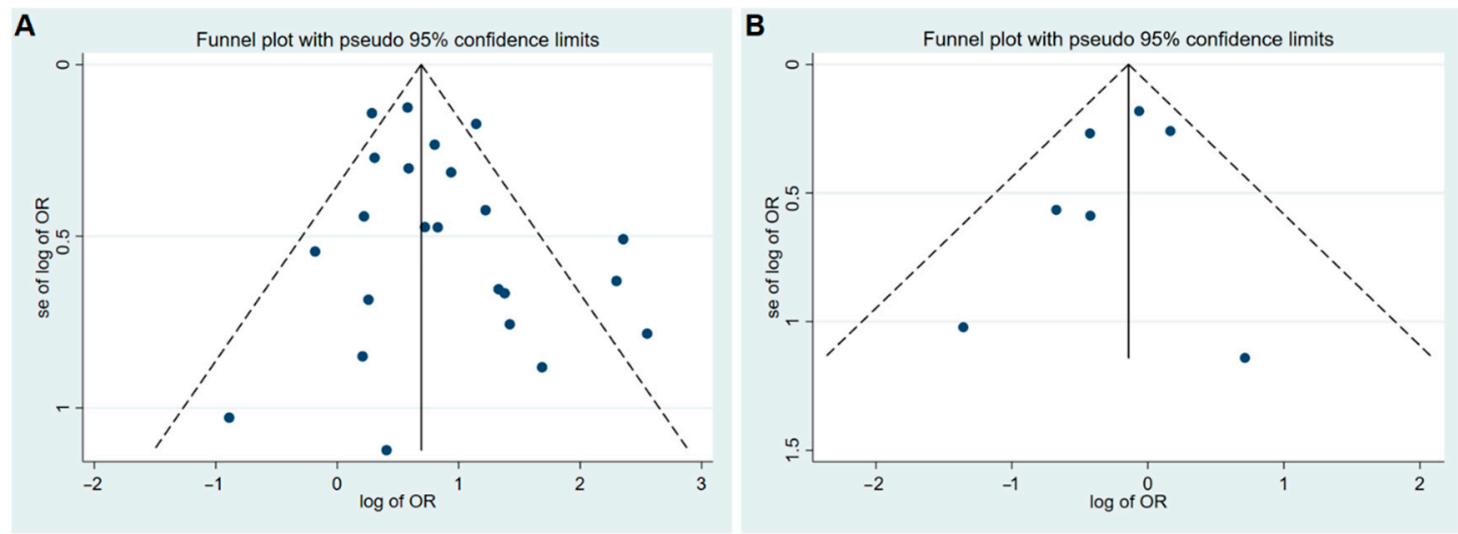

Figure A1. Funnel plots for (A) studies included in the COPD analyses; Egger's test $p=0.139$ and (B) studies included in the asthma analyses; Egger's test $p=0.388$.

Table A1. Full text articles excluded with reasons.

\begin{tabular}{ll}
\hline Exclusion Reasons & $n$ \\
\hline Studies that enrolled special populations (e.g., cancer patients) & 12 \\
\hline Studies that did not report separate data on the outcomes of interest & 28 \\
\hline Studies that did not specifically report data on COPD and/or asthma & 49 \\
\hline
\end{tabular}




\section{References}

1. WHO. Chronic Respiratory Diseases-Burden of COPD Cited 2020 August. Available online: https://www.who.int/respiratory/ copd/burden/en/ (accessed on 18 February 2021).

2. Prevention CDC. US Centers for Disease Control and Prevention People Who are at Higher Risk for Severe Illness: Center for Disease Control. Available online: https:/ / www.cdc.gov/coronavirus/2019-ncov/need-extra-precautions/people-at-higherrisk.html (accessed on 18 February 2021).

3. Villarroel, M.A.; Blackwell, D.L.; Jen, A. Adults: 2018 National Health Interview Survey. National Center for Health Statistics. Available online: http:/ / www.cdc.gov/nchs/nhis/SHS/tables.htm (accessed on 18 February 2021).

4. Lozano, R.; Naghavi, M.; Foreman, K.; Lim, S.; Shibuya, K.; Aboyans, V.; Abraham, J.; Adair, T.; Aggarwal, R.; Ahn, S.Y.; et al. Global and regional mortality from 235 causes of death for 20 age groups in 1990 and 2010: A systematic analysis for the Global Burden of Disease Study. Lancet 2012, 380, 2095-2128. [CrossRef]

5. Fireman, P. Understanding asthma pathophysiology. Allergy Asthma Proc. 2003, 24, 79-83. [PubMed]

6. Matsumoto, K.; Inoue, H. Viral infections in asthma and COPD. Respir. Investig. 2014, 52, 92-100. [CrossRef] [PubMed]

7. Nicholson, K.G.; Kent, J.; Ireland, D.C. Respiratory viruses and exacerbations of asthma in adults. BMJ 1993, 307, 982-986. [CrossRef]

8. Zheng, X.-Y.; Xu, Y.-J.; Guan, W.-J.; Lin, L.-F. Regional, age and respiratory-secretion-specific prevalence of respiratory viruses associated with asthma exacerbation: A literature review. Arch. Virol. 2018, 163, 845-853. [CrossRef]

9. Kwak, H.J.; Park, D.W.; Kim, J.E.; Koo, G.W.; Moon, J.-Y.; Sohn, J.W.; Shin, D.H.; Park, M.K.; Park, T.S.; Kim, T.H.; et al. Prevalence and Risk Factors of Respiratory Viral Infections in Exacerbations of Chronic Obstructive Pulmonary Disease. Tohoku J. Exp. Med. 2016, 240, 131-139. [CrossRef]

10. Althani, A.; Bushra, S.; Shaath, N.; Sattar, H.A. Characterisation of winter respiratory viral infections in patients with asthma and COPD in Qatar. Arch. Virol. 2012, 158, 1079-1083. [CrossRef] [PubMed]

11. Yormaz, B.; Findik, D.; Süerdem, M. Differences of viral panel positive versus negative by real-time PCR in COPD exacerbated patients. Tuberk. Toraks 2019, 67, 124-130. [CrossRef]

12. Zwaans, W.; Mallia, P.; van Winden, M.; Rohde, G. The relevance of respiratory viral infections in the exacerbations of chronic obstructive pulmonary disease-A systematic review. J. Clin. Virol. 2014, 61, 181-188. [CrossRef]

13. Liao, H.; Yang, Z.; Yang, C.; Tang, Y.; Liu, S.; Guan, W.G.; Chen, R.C. Impact of viral infection on acute exacerbation of asthma in out-patient clinics: A prospective study. J. Thorac. Dis. 2016, 8, 505-512. [CrossRef]

14. Atmar, R.L.; Guy, E.; Guntupalli, K.K.; Zimmerman, J.L.; Bandi, V.D.; Baxter, B.D.; Greenberg, S.B. Respiratory tract viral infections in inner-city asthmatic adults. Arch. Intern. Med. 1998, 158, 2453-2459. [CrossRef] [PubMed]

15. Kistler, A.; Avila, P.C.; Rouskin, S.; Wang, D.; Ward, T.; Yagi, S.; Schnurr, D.; Ganem, D.; DeRisi, J.L.; Boushey, H.A. Pan-Viral Screening of Respiratory Tract Infections in Adults With and Without Asthma Reveals Unexpected Human Coronavirus and Human Rhinovirus Diversity. J. Infect. Dis. 2007, 196, 817-825. [CrossRef]

16. Papadopoulos, N.G.; Christodoulou, I.; Rohde, G.; Agache, I.; Almqvist, C.; Bruno, A.; Bonini, S.; De Bont, L.G.M.; Bossios, A.; Bousquet, J.; et al. Viruses and bacteria in acute asthma exacerbations-A GA2LEN-DARE* systematic review. Allergy 2010, 66, 458-468. [CrossRef] [PubMed]

17. Fernandes-Matano, L.; Monroy-Muñoz, I.E.; Angeles-Martínez, J.; Sarquiz-Martinez, B.; Palomec-Nava, I.D.; Pardavé-Alejandre, H.D.; Coy-Arechavaleta, A.S.; Santacruz-Tinoco, C.E.; González-Ibarra, J.; González-Bonilla, C.R.; et al. Prevalence of noninfluenza respiratory viruses in acute respiratory infection cases in Mexico. PLoS ONE 2017, 12, e0176298. [CrossRef] [PubMed]

18. World Health Organization. WHO Coronavirus Disease (COVID-19) Dashboard. Available online: https:/ / covid19.who.int/ (accessed on 18 February 2021).

19. Moher, D.; Liberati, A.; Tetzlaff, J.; Altman, D.G.; The PRISMA Group. Preferred reporting items for systematic reviews and meta-analyses: The PRISMA statement. PLoS Med. 2009, 6, e1000097. [CrossRef]

20. Bullen, C.; Howe, C.; Laugesen, M.; McRobbie, H.; Parag, V.; Williman, J.; Walker, N. Electronic cigarettes for smoking cessation: A randomised controlled trial. Lancet 2013, 382, 1629-1637. [CrossRef]

21. Higgins, J.P.T.; Thompson, S.G. Quantifying heterogeneity in a meta-analysis. Stat. Med. 2002, 21, 1539-1558. [CrossRef] [PubMed]

22. Covino, M.; De Matteis, G.; Santoro, M.; Sabia, L.; Simeoni, B.; Candelli, M.; Ojetti, V.; Franceschi, F. Clinical characteristics and prognostic factors in COVID-19 patients aged $\geq 80$ years. Geriatr. Gerontol. Int. 2020, 20, 704-708. [CrossRef]

23. De La Rica, R.; Borges, M.; Aranda, M.; Del Castillo, A.; Socias, A.; Payeras, A.; Rialp, G.; Socias, L.; Masmiquel, L.; GonzalezFreire, M. Low Albumin Levels Are Associated with Poorer Outcomes in a Case Series of COVID-19 Patients in Spain: A Retrospective Cohort Study. Microorganisms 2020, 8, 1106. [CrossRef]

24. Di Bella, S.; Cesareo, R.; De Cristofaro, P.; Palermo, A.; Sanson, G.; Roman-Pognuz, E.; Zerbato, V.; Manfrini, S.; Giacomazzi, D.; Bo, E.D.; et al. Neck circumference as reliable predictor of mechanical ventilation support in adult inpatients with COVID-19: A multicentric prospective evaluation. Diabetes/Metab. Res. Rev. 2021, 37, e3354. [CrossRef]

25. Tambe, M.P.; Parande, M.; Tapare, V.S.; Borle, P.S.; Lakde, R.N.; Shelke, S.C.; BJMC COVID Epidemiology Group. An epidemiological study of laboratory confirmed COVID-19 cases admitted in a tertiary care hospital of Pune, Maharashtra. Indian J. Public Health 2020, 64, 183-S187. [CrossRef] 
26. Shahriarirad, R.; Khodamoradi, Z.; Erfani, A.; Hosseinpour, H.; Ranjbar, K.; Emami, Y.; Mirahmadizadeh, A.; Lotfi, M.; Yeganeh, B.S.; Nejad, A.D.; et al. Epidemiological and clinical features of 2019 novel coronavirus diseases (COVID-19) in the South of Iran. BMC Infect. Dis. 2020, 20, 427. [CrossRef] [PubMed]

27. Regina, J.; Papadimitriou-Olivgeris, M.; Burger, R.; Le Pogam, M.-A.; Niemi, T.; Filippidis, P.; Tschopp, J.; Desgranges, F.; Viala, B.; Kampouri, E.; et al. Epidemiology, risk factors and clinical course of SARS-CoV-2 infected patients in a Swiss university hospital: An observational retrospective study. PLoS ONE 2020, 15, e0240781. [CrossRef] [PubMed]

28. Satici, C.; Demirkol, M.A.; Altunok, E.S.; Gursoy, B.; Alkan, M.; Kamat, S.; Demirok, B.; Surmeli, C.D.; Calik, M.; Cavus, Z.; et al. Performance of pneumonia severity index and CURB-65 in predicting 30-day mortality in patients with COVID-19. Int. J. Infect. Dis. 2020, 98, 84-89. [CrossRef]

29. Lee, L.Y.W.; Cazier, J.-B.; Angelis, V.; Arnold, R.; Bisht, V.; Campton, N.A.; Chackathayil, J.; Cheng, V.W.; Curley, H.M.; Fittall, M.W.; et al. COVID-19 mortality in patients with cancer on chemotherapy or other anticancer treatments: A prospective cohort study. Lancet 2020, 395, 1919-1926. [CrossRef]

30. Argenziano, M.G.; Bruce, S.L.; Slater, C.L.; Tiao, J.R.; Baldwin, M.R.; Barr, R.G.; Chang, B.P.; Chau, K.H.; Choi, J.J.; Gavin, N.; et al. Characterization and clinical course of 1000 patients with coronavirus disease 2019 in New York: Retrospective case series. BMJ 2020, 369, m1996. [CrossRef] [PubMed]

31. Imam, Z.; Odish, F.; Gill, I.; O'Connor, D.; Armstrong, J.; Vanood, A.; Ibironke, O.; Hanna, A.; Ranski, A.; Halalau, A. Older age and comorbidity are independent mortality predictors in a large cohort of 1305 COVID-19 patients in Michigan, United States. J. Intern. Med. 2020, 288, 469-476. [CrossRef] [PubMed]

32. Nowak, B.; Szymański, P.; Pańkowski, I.; Szarowska, A.; Życińska, K.; Rogowski, W.; Gil, R.; Furmanek, M.; Tatur, J.; Zaczyński, A.; et al. Clinical characteristics and short-term outcomes of coronavirus disease 2019: Retrospective, single-center experience of designated hospital in Poland. Pol. Arch. Intern. Med. 2020, 130, 407-411. [CrossRef]

33. Okoh, A.K.; Sossou, C.; Dangayach, N.S.; Meledathu, S.; Phillips, O.; Raczek, C.; Patti, M.; Kang, N.; Hirji, S.A.; Cathcart, C.; et al. Coronavirus disease 19 in minority populations of Newark, New Jersey. Int. J. Equity Health 2020, 19, 93. [CrossRef] [PubMed]

34. Palaiodimos, L.; Kokkinidis, D.G.; Li, W.; Karamanis, D.; Ognibene, J.; Arora, S.; Southern, W.N.; Mantzoros, C.S. Severe obesity, increasing age and male sex are independently associated with worse in-hospital outcomes, and higher in-hospital mortality, in a cohort of patients with COVID-19 in the Bronx, New York. Metabilism 2020, 108, 154262. [CrossRef]

35. Rentsch, C.T.; Kidwai-Khan, F.; Tate, J.P.; Park, L.S.; King, J.T., Jr.; Skanderson, M.; Hauser, R.G.; Schultze, A.; Jarvis, C.I.; Ho-lodniy, M.; et al. Covid-19 Testing, Hospital Admission, and Intensive Care among 2,026,227 United States Veterans Aged 54-75 Years. medRxiv 2020. [CrossRef]

36. Russo, V.; Di Maio, M.; Attena, E.; Silverio, A.; Scudiero, F.; Celentani, D.; Lodigiani, C.; Di Micco, P. Clinical impact of preadmission antithrombotic therapy in hospitalized patients with COVID-19: A multicenter observational study. Pharmacol. Res. 2020, 159, 104965. [CrossRef] [PubMed]

37. Pérez-Tanoira, R.; Pérez-García, F.; Romanyk, J.; Gómez-Herruz, P.; Arroyo, T.; González, R.; García, L.L.; Expósito, C.V.; Moreno, J.S.; Gutiérrez, I.; et al. Prevalence and risk factors for mortality related to COVID-19 in a severely affected area of Madrid, Spain. medRxiv 2020. [CrossRef]

38. Foy, B.H.; Carlson, J.C.; Reinertsen, E.; Valls, R.P.; Lopez, R.P.; Palanques-Tost, E.; Mow, C.; Westover, M.B.; Aguirre, A.D.; Higgins, J.M. Elevated RDW is Associated with Increased Mortality Risk in COVID-19. medRxiv 2020. [CrossRef]

39. Li, L.; Yang, L.; Gui, S.; Pan, F.; Ye, T.; Liang, B.; Hu, Y.; Zheng, C. Association of clinical and radiographic findings with the outcomes of 93 patients with COVID-19 in Wuhan, China. Theranostics 2020, 10, 6113-6121. [CrossRef]

40. Liu, Y.; Du, X.; Chen, J.; Jin, Y.; Peng, L.; Wang, H.H.; Luo, M.; Chen, L.; Zhao, Y. Neutrophil-to-lymphocyte ratio as an independent risk factor for mortality in hospitalized patients with COVID-19. J. Infect. 2020, 81, e6-e12. [CrossRef]

41. Javanian, M.; Bayani, M.; Shokri, M.; Sadeghi-Haddad-Zavareh, M.; Babazadeh, A.; Yeganeh, B.; Mohseni, S.; Mehraein, R.; Sepidarkish, M.; Bijani, A.; et al. Clinical and laboratory findings from patients with COVID-19 pneumonia in Babol North of Iran: A retrospective cohort study. Roman. J. Intern. Med. 2020, 58, 161-167. [CrossRef]

42. Zhou, F.; Yu, T.; Du, R.; Fan, G.; Liu, Y.; Liu, Z.; Xiang, J.; Wang, Y.; Song, B.; Gu, X.; et al. Clinical course and risk factors for mortality of adult inpatients with COVID-19 in Wuhan, China: A retrospective cohort study. Lancet 2020, 395, 1054-1062. [CrossRef]

43. Gao, C.; Cai, Y.; Zhang, K.; Zhou, L.; Zhang, Y.; Zhang, X.; Li, Q.; Li, W.; Yang, S.; Zhao, X.; et al. Association of hypertension and antihypertensive treatment with COVID-19 mortality: A retrospective observational study. Eur. Hear. J. 2020, 41, $2058-2066$. [CrossRef]

44. Huang, J.; Cheng, A.; Kumar, R.; Fang, Y.; Chen, G.; Zhu, Y.; Lin, S. Hypoalbuminemia predicts the outcome of COVID-19 independent of age and co-morbidity. J. Med. Virol. 2020, 92, 2152-2158. [CrossRef] [PubMed]

45. Urra, J.; Cabrera, C.; Porras, L.; Ródenas, I. Selective CD8 cell reduction by SARS-CoV-2 is associated with a worse prognosis and systemic inflammation in COVID-19 patients. Clin. Immunol. 2020, 217, 108486. [CrossRef]

46. Xu, P.P.; Tian, R.H.; Luo, S.; Zu, Z.Y.; Fan, B.; Wang, X.M.; Xu, K.; Wang, J.T.; Zhu, J.; Shi, J.C.; et al. Risk factors for adverse clinical outcomes with COVID-19 in China: A multicenter, retrospective, observational study. Theranostics 2020, 10, 6372-6383. [CrossRef] [PubMed] 
47. Auld, S.C.; Caridi-Scheible, M.; Blum, J.M.; Robichaux, C.; Kraft, C.; Jacob, J.T.; Jabaley, C.S.; Carpenter, D.; Kaplow, R.; HernandezRomieu, A.C.; et al. ICU and Ventilator Mortality Among Critically Ill Adults with Coronavirus Disease. Crit. Care Med. 2020. Publish Ahead. [CrossRef] [PubMed]

48. Borobia, A.M.; Carcas, A.J.; Arnalich, F.; Álvarez-Sala, R.; Monserrat-Villatoro, J.; Quintana, M.; Figueira, J.C.; Santos-Olmo, R.M.T.; García-Rodríguez, J.; Martín-Vega, A.; et al. A Cohort of Patients with COVID-19 in a Major Teaching Hospital in Europe. J. Clin. Med. 2020, 9, 1733. [CrossRef] [PubMed]

49. Cariou, B.; Hadjadj, S.; Wargny, M.; Pichelin, M.; Al-Salameh, A.; Allix, I.; Amadou, C.; Arnault, G.; Baudoux, F.; The CORONADO Investigators; et al. Phenotypic characteristics and prognosis of inpatients with COVID-19 and diabetes: The CORONADO study. Diabetologia 2020, 63, 1500-1515. [CrossRef] [PubMed]

50. Murillo-Zamora, E.; Hernandez-Suarez, C.M. Survival in adult inpatients with COVID-19. Public Health 2021, 190, 1-3. [CrossRef]

51. Israelsen, S.B.; Kristiansen, K.T.; Hindsberger, B.; Ulrik, C.S.; Andersen, O.; Jensen, M.; Andersen, S.; Rasmussen, C.; Jørgensen, H.L.; Østergaard, C.; et al. Characteristics of patients with COVID-19 pneumonia at Hvidovre Hospital, March-April 2020. Dan. Med. J. 2020, 67, A05200313.

52. Alqahtani, J.S.; Oyelade, T.; Aldhahir, A.M.; Alghamdi, S.M.; Almehmadi, M.; Alqahtani, A.S.; Quaderi, S.; Mandal, S.; Hurst, J.R. Prevalence, Severity and Mortality associated with COPD and Smoking in patients with COVID-19: A Rapid Systematic Review and Meta-Analysis. PLoS ONE 2020, 15, e0233147. [CrossRef]

53. Petrilli, C.M.; Jones, S.; Yang, J.; Rajagopalan, H.; O’Donnell, L.; Chernyak, Y.; Tobin, K.; Cerfolio, R.J.; Francois, F.; Horwitz, L. Factors associated with hospital admission and critical illness among 5279 people with coronavirus disease 2019 in New York City: Prospective cohort study. BMJ 2020,369, m1966. [CrossRef]

54. Tartof, S.Y.; Qian, L.; Hong, M.V.; Wei, M.R.; Nadjafi, R.F.; Fischer, H.; Li, M.Z.; Shaw, D.S.F.; Caparosa, M.S.L.; Nau, C.L.; et al. Obesity and Mortality Among Patients Diagnosed With COVID-19: Results From an Integrated Health Care Organization. Ann. Intern. Med. 2020, 173, 773-781. [CrossRef]

55. Lücker, L.M.; Kherad, O.; Iten, A.; Wagner, N.; Descombes, M.; Camus, V.; Kaiser, L.; Louis-Simonet, M. Clinical features and outcome of hospitalised adults and children with the 2009 influenza A H1N1 infection at Geneva's University Hospital. Swiss Med. Wkly. 2011, 141. [CrossRef] [PubMed]

56. Bouneb, R.; Mellouli, M.; Bensoltane, H.; Baroudi, J.; Chouchene, I.; Boussarsar, M. Characteristics and outcome of ill critical patients with influenza a infection. Pan Afr. Med. J. 2018, 29, 1-8. [CrossRef] [PubMed]

57. Thompson, W.W.; Shay, D.K.; Weintraub, E.; Brammer, L.; Cox, N.; Anderson, L.J.; Fukuda, K. Mortality Associated with Influenza and Respiratory Syncytial Virus in the United States. JAMA 2003, 289, 179-186. [CrossRef]

58. Kim, H.-C.; Choi, S.-H.; Huh, J.-W.; Sung, H.; Hong, S.B.; Lim, C.-M.; Koh, Y. Different pattern of viral infections and clinical outcomes in patient with acute exacerbation of chronic obstructive pulmonary disease and chronic obstructive pulmonary disease with pneumonia. J. Med. Virol. 2016, 88, 2092-2099. [CrossRef]

59. Bramley, A.M.; Dasgupta, S.; Skarbinski, J.; Kamimoto, L.; Fry, A.M.; Finelli, L.; Jain, S. Intensive care unit patients with 2009 pandemic influenza A (H1N1pdm09) virus infection-United States. Influ. Other Respir. Viruses 2012, 6, e134-e142. [CrossRef] [PubMed]

60. Cheng, A.C.; Kotsimbos, T.; Reynolds, A.; Bowler, S.D.; Brown, S.G.; Hancox, R.J.; Holmes, M.; Irving, L.; Jenkins, C.; Thompson, P.; et al. Clinical and epidemiological profile of patients with severe H1N1/09 pandemic influenza in Australia and New Zealand: An observational cohort study. BMJ Open 2011, 1, e000100. [CrossRef] [PubMed]

61. Jamaati, H.; Mohajerani, S.A.; Shamaee, M.; Chitsazan, M.; Radmand, G.; Maadani, M.; Mansouri, S.D.; Hashemian, S.M.R.; Tabarsi, P.; Nadji, S.A. Secondary infection and clinical aspects after pandemic swine-origin influenza a (H1N1) admission in an Iranian critical care unite. Int. J. Crit. Illn. Inj. Sci. 2014, 4, 309-313. [CrossRef]

62. Jaber, S.; Conseil, M.; Coisel, Y.; Jung, B.; Chanques, G. Grippe A (H1N1) et SDRA: Caractéristiques des patients admis en réanimation et prise en charge. Revue de la littérature. Ann. Fr. d'Anesth. Réanim. 2010, 29, 117-125. [CrossRef]

63. Fabbiani, M.; Sali, M.; Di Cristo, V.; Pignataro, G.; Prete, V.; Farina, S.; D’Avino, A.; Manzara, S.; Verme, L.Z.D.; Silveri, N.G.; et al. Prospective evaluation of epidemiological, clinical, and microbiological features of pandemic influenza A (H1N1) virus infection in Italy. J. Med. Virol. 2011, 83, 2057-2065. [CrossRef]

64. Peralta, P.S.-O.; Cortes-García, M.; Vicente-Herrero, M.; Castrillo-Villamandos, C.; Arias-Bohigas, P.; Amo, I.P.-D.; Sierra-Moros, M.J.; on behalf of the Surveillance Group for New Influenza A(H1N1) Virus Investigation and Control Team in Spain. Risk factors for disease severity among hospitalised patients with 2009 pandemic influenza A (H1N1) in Spain, April-December 2009. Eurosurveillance 2010, 15, 19667. [CrossRef]

65. Booth, C.M.; Matukas, L.M.; Tomlinson, G.A.; Rachlis, A.R.; Rose, D.B.; Dwosh, H.A.; Walmsley, S.L.; Mazzulli, T.; Avendano, M.; Derkach, P.; et al. Clinical Features and Short-term Outcomes of 144 Patients With SARS in the Greater Toronto Area. JAMA 2003, 289, 2801-2809. [CrossRef]

66. Leung, G.M.; Hedley, A.J.; Ho, L.-M.; Chau, P.; Wong, I.O.; Thach, T.Q.; Ghani, A.C.; Donnelly, C.A.; Fraser, C.; Riley, S.; et al. The Epidemiology of Severe Acute Respiratory Syndrome in the 2003 Hong Kong Epidemic: An Analysis of All 1755 Patients. Ann. Intern. Med. 2004, 141, 662-673. [CrossRef] [PubMed]

67. Lu, W.; Zhang, H.S.; Wang, F.M.; Wang, S.J.; Wu, X.D.; Zhang, N.X.; Kan, Z.C.; Qin, Y.Z.; Xiao, L. Preliminary analysis of treatment in 32 patients with critical severe acute respiratory syndrome. Zhongguo Wei Zhong Bing Ji Jiu Yi Xue 2003, 15, 492-494. [PubMed] 
68. Rohde, G.; Borg, I.; Arinir, U.; Dorsten, C.; Schultze-Werninghaus, G.; Bauer, T. T Evaluation of a real-time polymerase-chain reaction for severe acute respiratory syndrome (SARS) associated coronavirus in patients with hospitalised exacerbation of COPD. Eur. J. Med. Res. 2004, 9, 505-509. [PubMed]

69. Kherad, O.; Kaiser, L.; Bridevaux, P.-O.; Sarasin, F.; Thomas, Y.; Janssens, J.-P.; Rutschmann, O.T. Upper-Respiratory Viral Infection, Biomarkers, and COPD Exacerbations. Chest 2010, 138, 896-904. [CrossRef]

70. Radzikowska, U.; Ding, M.; Tan, G.; Zhakparov, D.; Peng, Y.; Wawrzyniak, P.; Wang, M.; Li, S.; Morita, H.; Altunbulakli, C.; et al. Distribution of ACE2, CD147, CD26, and other SARS-CoV-2 associated molecules in tissues and immune cells in health and in asthma, COPD, obesity, hypertension, and COVID-19 risk factors. Allergy 2020, 75, 2829-2845. [CrossRef]

71. Gu, J.; Gong, E.; Zhang, B.; Zheng, J.; Gao, Z.; Zhong, Y.; Zou, W.; Zhan, J.; Wang, S.; Xie, Z.; et al. Multiple organ infection and the pathogenesis of SARS. J. Exp. Med. 2005, 202, 415-424. [CrossRef]

72. Hoffmann, M.; Kleine-Weber, H.; Schroeder, S.; Krüger, N.; Herrler, T.; Erichsen, S.; Schiergens, T.S.; Herrler, G.; Wu, N.-H.; Nitsche, A.; et al. SARS-CoV-2 Cell Entry Depends on ACE2 and TMPRSS2 and Is Blocked by a Clinically Proven Protease Inhibitor. Cell 2020, 181, 271-280. [CrossRef]

73. Cheng, Z.; Zhou, J.; To, K.K.-W.; Chu, H.; Li, C.; Wang, D.; Yang, D.; Zheng, S.; Hao, K.; Bossé, Y.; et al. Identification ofTMPRSS2as a Susceptibility Gene for Severe 2009 Pandemic A(H1N1) Influenza and A(H7N9) Influenza. J. Infect. Dis. 2015, 212, 1214-1221. [CrossRef]

74. Li, W.; Moore, M.J.; Vasilieva, N.; Sui, J.; Wong, S.K.; Berne, M.A.; Somasundaran, M.; Sullivan, J.L.; Luzuriaga, K.; Greenough, T.C.; et al. Angiotensin-converting enzyme 2 is a functional receptor for the SARS coronavirus. Nature 2003, 426, 450-454. [CrossRef]

75. Brake, S.J.; Barnsley, K.; Lu, W.; McAlinden, K.D.; Eapen, M.S.; Sohal, S.S. Smoking Upregulates Angiotensin-Converting Enzyme-2 Receptor: A Potential Adhesion Site for Novel Coronavirus SARS-CoV-2 (Covid-19). J. Clin. Med. 2020, 9, 841. [CrossRef]

76. Leung, J.M.; Yang, C.X.; Tam, A.; Shaipanich, T.; Hackett, T.-L.; Singhera, G.K.; Dorscheid, D.R.; Sin, D.D. ACE-2 expression in the small airway epithelia of smokers and COPD patients: Implications for COVID-19. Eur. Respir. J. 2020, 55, 2000688. [CrossRef]

77. Rossato, M.; Russo, L.; Mazzocut, S.; Di Vincenzo, A.; Fioretto, P.; Vettor, R. Current smoking is not associated with COVID-19. Eur. Respir. J. 2020, 55, 2001290. [CrossRef] [PubMed]

78. Guan, W.J.; Liang, W.H.; Zhao, Y.; Liang, H.-R.; Chen, Z.-S.; Li, Y.-M.; Liu, X.-Q.; Chen, R.-C.; Tang, C.-L.; Wang, T.; et al. Comorbidity and its impact on 1590 patients with COVID-19 in China: A nationwide analysis. Eur. Respir. J. 2020, 55, 2000547. [CrossRef] [PubMed]

79. Sharif-Askari, N.S.; Sharif-Askari, F.S.; Alabed, M.; Temsah, M.-H.; Al Heialy, S.; Hamid, Q.; Halwani, R. Airways Expression of SARS-CoV-2 Receptor, ACE2, and TMPRSS2 Is Lower in Children Than Adults and Increases with Smoking and COPD. Mol. Ther. Methods Clin. Dev. 2020, 18, 1-6. [CrossRef]

80. Kim, W.J.; Lim, J.H.; Lee, J.S.; Lee, S.-D.; Kim, J.H.; Oh, Y.-M. Comprehensive Analysis of Transcriptome Sequencing Data in the Lung Tissues of COPD Subjects. Int. J. Genom. 2015, 2015, 1-9. [CrossRef] [PubMed]

81. Pinto, B.G.G.; Oliveira, A.E.R.; Singh, Y.; Jimenez, L.; Gonçalves, A.N.; Ogava, R.L.T.; Creighton, R.; Peron, J.P.S.; Nakaya, H.I. ACE2 Expression Is Increased in the Lungs of Patients with Comorbidities Associated with Severe COVID-19. J. Infect. Dis. 2020, 222, 556-563. [CrossRef] [PubMed]

82. Smith, J.C.; Sausville, E.L.; Girish, V.; Yuan, M.L.; Vasudevan, A.; John, K.M.; Sheltzer, J.M. Cigarette Smoke Exposure and Inflammatory Signaling Increase the Expression of the SARS-CoV-2 Receptor ACE2 in the Respiratory Tract. Dev. Cell 2020, 53, 514-529.e3. [CrossRef]

83. Matusiak, M.; Schürch, C.M. Expression of SARS-CoV-2 entry receptors in the respiratory tract of healthy individuals, smokers and asthmatics. Respir. Res. 2020, 21, 1-6. [CrossRef]

84. Song, J.; Zeng, M.; Wang, H.; Qin, C.; Hou, H.; Sun, Z.; Xu, S.; Wang, G.; Guo, C.; Deng, Y.; et al. Distinct effects of asthma and COPD comorbidity on disease expression and outcome in patients with COVID-19. Allergy 2021, 76, 483-496. [CrossRef]

85. Drummond, M.B.; Wise, R.A.; Hansel, N.N.; Putcha, N. Comorbidities and Chronic Obstructive Pulmonary Disease: Prevalence, Influence on Outcomes, and Management. Semin. Respir. Crit. Care Med. 2015, 36, 575-591. [CrossRef] [PubMed]

86. Chen, W.; Thomas, J.; Sadatsafavi, M.; FitzGerald, J.M. Risk of cardiovascular comorbidity in patients with chronic obstructive pulmonary disease: A systematic review and meta-analysis. Lancet Respir. Med. 2015, 3, 631-639. [CrossRef]

87. Corne, J.M.; Marshall, C.; Smith, S.; Schreiber, J.; Sanderson, G.; Holgate, S.T.; Johnston, S.L. Frequency, severity, and duration of rhinovirus infections in asthmatic and non-asthmatic individuals: A longitudinal cohort study. Lancet 2002, 359, 831-834. [CrossRef]

88. Lovinsky-Desir, S.; Deshpande, D.R.; De, A.; Murray, L.; Stingone, J.A.; Chan, A.; Patel, N.; Rai, N.; DiMango, E.; Milner, J.; et al. Asthma among hospitalized patients with COVID-19 and related outcomes. J. Allergy Clin. Immunol. 2020, 146, 1027-1034.e4. [CrossRef]

89. Chhiba, K.D.; Patel, G.B.; Vu, T.H.T.; Chen, M.M.; Guo, A.; Kudlaty, E.; Mai, Q.; Yeh, C.; Muhammad, L.N.; Harris, K.E.; et al. Prevalence and characterization of asthma in hospitalized and nonhospitalized patients with COVID-19. J. Allergy Clin. Immunol. 2020, 146, 307-314.e4. [CrossRef] 
90. Avdeev, S.; Moiseev, S.; Brovko, M.; Yavorovskiy, A.; Umbetova, K.; Akulkina, L.; Tsareva, N.; Merzhoeva, Z.; Gainitdinova, V.; Fomin, V. Low prevalence of bronchial asthma and chronic obstructive lung disease among intensive care unit patients with COVID-19. Allergy 2020, 75, 2703-2704. [CrossRef] [PubMed]

91. Lieberman-Cribbin, W.; Rapp, J.; Alpert, N.; Tuminello, S.; Taioli, E. The Impact of Asthma on Mortality in Patients with COVID-19. Chest 2020, 158, 2290-2291. [CrossRef] [PubMed]

92. Klemets, P.; Lyytikäinen, O.; Ruutu, P.; Ollgren, J.; Kaijalainen, T.; Leinonen, M.; Nuorti, J.P. Risk of invasive pneumococcal infections among working age adults with asthma. Thorax 2010, 65, 698-702. [CrossRef]

93. Talbot, T.R.; Hartert, T.V.; Mitchel, E.; Halasa, N.B.; Arbogast, P.G.; Poehling, K.A.; Schaffner, W.; Craig, A.S.; Griffin, M.R. Asthma as a Risk Factor for Invasive Pneumococcal Disease. N. Engl. J. Med. 2005, 352, 2082-2090. [CrossRef]

94. Black, P.N.; Blasi, F.; Jenkins, C.R.; Scicchitano, R.; Mills, G.D.; Rubinfeld, A.R.; Ruffin, R.E.; Mullins, P.R.; Dangain, J.; Cooper, B.C.; et al. Trial of Roxithromycin in Subjects with Asthma and Serological Evidence of Infection with Chlamydia pneumoniae. Am. J. Respir. Crit. Care Med. 2001, 164, 536-541. [CrossRef]

95. Busse, W.W.; Lemanske, R.F.; Gern, J. Role of viral respiratory infections in asthma and asthma exacerbations. Lancet 2010, 376, 826-834. [CrossRef]

96. Grissell, T.V.; Powell, H.; Shafren, D.R.; Boyle, M.J.; Hensley, M.J.; Jones, P.D.; Whitehead, B.F.; Gibson, P.G. Interleukin-10 Gene Expression in Acute Virus-induced Asthma. Am. J. Respir. Crit. Care Med. 2005, 172, 433-439. [CrossRef] [PubMed]

97. Lee, S.W.; Lee, S.; Sheen, Y.H.; Ha, E.K.; Choi, S.H.; Yang, M.-S.; Hwang, S.; Kim, S.S.; Choi, J.-H.; Han, M.Y. Seasonal Cycle and Relationship of Seasonal Rhino- and Influenza Virus Epidemics With Episodes of Asthma Exacerbation in Different Age Groups. Allergy Asthma Immunol. Res. 2017, 9, 517-525. [CrossRef] [PubMed]

98. Trinh, P.; Jung, T.H.; Keene, D.; Demmer, R.T.; Perzanowski, M.; Lovasi, G. Temporal and spatial associations between influenza and asthma hospitalisations in New York City from 2002 to 2012: A longitudinal ecological study. BMJ Open 2018, 8, e020362. [CrossRef]

99. Morales-Suárez-Varela, M.; Llopis-González, A.; Vergara-Hernández, C.; Fernandez-Fabrellas, E.; Sanz, F.; Perez-Lozano, M.J.; Martin, V.; Astray, J.; Castilla, J.; Egurrola, M.; et al. Asthma in older people hospitalized with influenza in Spain: A case-control study. Allergy Asthma Proc. 2017, 38, 277-285. [CrossRef] [PubMed]

100. Feldman, L.Y.; Zhu, J.; To, T. Estimating age-specific influenza-associated asthma morbidity in Ontario, Canada. Respir. Med. 2019, 155, 104-112. [CrossRef] [PubMed]

101. Nguyen-Van-Tam, J.S.; Openshaw, P.J.M.; Hashim, A.; Gadd, E.M.; Lim, W.S.; Semple, M.G.; Read, R.C.; Taylor, B.L.; Brett, S.J.; McMenamin, J.; et al. Risk factors for hospitalisation and poor outcome with pandemic A/H1N1 influenza: United Kingdom first wave (May-September 2009). Thorax 2010, 65, 645-651. [CrossRef]

102. Rodríguez-Rieiro, C.; Carrasco-Garrido, P.; Hernandez-Barrera, V.; De Andres, A.L.; Jimenez-Trujillo, I.; Gil De Miguel, Á.; Jimenez-Garcia, R. Pandemic influenza hospitalization in Spain (2009): Incidence, in-hospital mortality, comorbidities and costs. Hum. Vaccines Immunother. 2012, 8, 443-447. [CrossRef]

103. Kim, M.H.; Song, W.J.; Yang, M.S.; Lee, S.H.; Kwon, J.W.; Kim, S.H.; Kang, H.R.; Park, H.W.; Cho, Y.J.; Cho, S.H.; et al. Clinical course of asthma patients with H1N1 influenza infection and oseltamivir. Minerva Med. 2018, 109, 7-14. [CrossRef]

104. Dhawale, V.S.; Amara, V.R.; Karpe, P.A.; Malek, V.; Patel, D.; Tikoo, K. Activation of angiotensin-converting enzyme 2 (ACE2) attenuates allergic airway inflammation in rat asthma model. Toxicol. Appl. Pharmacol. 2016, 306, 17-26. [CrossRef] [PubMed]

105. Camiolo, M.; Gauthier, M.; Kaminski, N.; Ray, A.; Wenzel, S.E. Expression of SARS-CoV-2 receptor ACE2 and coincident host response signature varies by asthma inflammatory phenotype. J. Allergy Clin. Immunol. 2020, 146, 315-324.e7. [CrossRef] [PubMed]

106. Jackson, D.J.; Busse, W.W.; Bacharier, L.B.; Kattan, M.; O'Connor, G.T.; Wood, R.A.; Visness, C.M.; Durham, S.R.; Larson, D.; Esnault, S.; et al. Association of respiratory allergy, asthma, and expression of the SARS-CoV-2 receptor ACE2. J. Allergy Clin. Immunol. 2020, 146, 203-206.e3. [CrossRef] [PubMed]

107. Kimura, H.; Francisco, D.; Conway, M.; Martinez, F.D.; Vercelli, D.; Polverino, F.; Billheimer, D.; Kraft, M. Type 2 inflammation modulates ACE2 and TMPRSS2 in airway epithelial cells. J. Allergy Clin. Immunol. 2020, 146, 80-88.e8. [CrossRef] [PubMed]

108. Gershon, A.S.; Thiruchelvam, D.; Chapman, K.R.; Aaron, S.D.; Stanbrook, M.B.; Bourbeau, J.; Tan, W.; To, T. Health Services Burden of Undiagnosed and Overdiagnosed COPD. Chest 2018, 153, 1336-1346. [CrossRef] 